\title{
Searching for energy-resolved quasi-periodic oscillations in AGN
}

\author{
Dominic I. Ashton, ${ }^{1 \star} \&$ Matthew J. Middleton ${ }^{1}$ \\ ${ }^{1}$ Department of Physics \& Astronomy, University of Southampton, Southampton, SO17 1BJ, UK
}

Accepted XXX. Received YYY; in original form ZZZ

\begin{abstract}
X-ray quasi-periodic oscillations (QPOs) in AGN allow us to probe and understand the nature of accretion in highly curved space-time, yet the most robust form of detection (i.e. repeat detections over multiple observations) has been limited to a single source to-date, with only tentative claims of single observation detections in several others. The association of those established AGN QPOs with a specific spectral component has motivated us to search the $X M M$-Newton archive and analyse the energy-resolved lightcurves of 38 bright AGN. We apply a conservative false alarm testing routine folding in the uncertainty and covariance of the underlying broad-band noise. We also explore the impact of red-noise leak and the assumption of various different forms (power-law, broken power-law and lorentzians) for the underlying broad-band noise. In this initial study, we report QPO candidates in 6 AGN (7 including one tentative detection in MRK 766) from our sample of 38, which tend to be found at characteristic energies and, in four cases, at the same frequency across at least two observations, indicating they are highly unlikely to be spurious in nature.
\end{abstract}

Key words: methods: data analysis - methods: statistical - galaxies: active - galaxies: Seyfert - X-rays: galaxies

\section{INTRODUCTION}

The accretion of gas onto black holes provides insights into the behaviour of matter in the strong gravity regime and can therefore test general relativity (GR) itself. As the X-rays trace the innermost regions (the disc and corona in black hole binaries - BHBs, and the corona in AGN) it is the rapid variability in this band which encodes the most valuable diagnostic information. Such studies have proven capable of disentangling the geometry of the flow (Fabian et al. 2009; Kara et al. 2019; Wilkins \& Fabian 2013; Alston et al. 2020a) whilst quasi-periodic oscillations (QPOs), ubiquitous in BHBs (Motta 2016) can provide information about the dynamics of the disc and the effects of external torques (e.g. the Lense-Thirring effect: Stella et al. 1999; Fragile et al. 2007; Ingram \& Done 2011).

Discovering QPOs in AGN has been of importance for some time, as they provide tests for the scalability of the accretion flow by black hole mass (e.g. McHardy et al. 2006), and a 'slow-motion' view of the process creating them (i.e. many more photons per QPO phase bin relative to BHBs). QPOs detected in BHBs (with masses $\sim 10 \mathrm{M}_{\odot}$ ) range from $1-100 \mathrm{~s}$ (the low frequency QPOs - LFQPOs), to $0.01-0.1 \mathrm{~s}$ (the high frequency QPOs - HFQPOs, see Remillard $\&$ McClintock 2006). Assuming a linear scaling to SMBH masses of $\sim 10^{6-7} \mathrm{M}_{\odot}$, implies AGN QPOs should have periods of days to weeks for LFQPO analogues, and hundreds of seconds to hours for the HFQPOs. It is therefore reasonable to speculate that, should a

^ E-mail: d.i.ashton@ soton.ac.uk simple scaling hold, the latter should be present within some long observations of AGN taken by existing X-ray instruments.

To-date there have been numerous claims of X-ray QPOs in AGN, although only a very small number have proven to be statistically robust upon detailed investigation. The first widely accepted detection was in the Narrow Line Seyfert 1 (NLS1), RE J1034+396 (Gierlinski et al. 2008) with a QPO period of approximately one hour, thereby resembling a mass-scaled BHB HFQPO (Middleton \& Done 2010). This QPO has been speculated to originate in the hardest energy spectral component, similar to the $67 \mathrm{~Hz}$ QPO of GRS 1915+105 (Middleton et al. 2009, 2011), and has remained detectable at around the same frequency across several observations (Alston et al. 2014, Jin et al. 2020). The chance probability of making a false signal appear across multiple observations at the same frequency is extremely low, and so the repeat detection of the QPO in RE J1034+396 lends strength to its identification as a bona-fide signal. In addition to RE J1034+396, there has been a QPO detection claim in a single observation MS 2254.9-3712 (Alston et al. 2015) with a period of $\sim 2$ hours, and reports of possible QPOs following different methodolgies in 1H 0707-495, MRK 766, IRAS 132243809 and ARK 564 (McHardy et al. 2007; Pan et al. 2016; Zhang et al. 2017, Zhang et al. 2018; Alston et al. 2019) (as well as in tidal disruption events, e.g. Pasham et al. 2019a). The confirmation of tentative QPO claims and the discovery of new AGN QPOs is important; the present sample size of robust detections is extremely small which hinders a rigorous analysis of their origin, and critiques of their similarities or differences when compared to BHB QPOs.

Central to the detection of a QPO is a statistical method which 
robustly tests the null hypothesis, and accounts for the shape of the underlying broad-band noise onto which the QPO is imprinted (Vaughan 2005, 2010; Vaughan et al. 2016). Previous large-scale efforts include González-Martín \& Vaughan (2012) who studied a large sample of $104 \mathrm{AGN}$ and described the properties of the high frequency noise en-masse but without locating new QPOs. Here we build on previous work by searching for QPOs in energyresolved $\mathrm{AGN}$ lightcurves, and present the discovery of several new statistically significant QPO candidates.

The paper is laid out as follows. In Section 2 we introduce the AGN sample and our methods of data reduction for these observations. In Section 3 we outline the statistical framework for our QPO search, including the tests we apply to probe any candidates in greater detail. In Section 4 we outline the results of the QPO search. In Section 5 we discuss the additional uncertainty in the broad-band noise and its possible impact on QPO detections. Lastly, we discuss the relevance and impact of our findings in Section 6.

\section{OBSERVATIONS}

\subsection{AGN Sample}

We construct our AGN sample primarily from the Palomar Green QSO (PGQSO) catalogue used by Crummy et al. (2006) and Middleton et al. (2007) as these are extremely bright sources and have been well-studied by XMM-Newton; this provides high signal-tonoise ratio data and the possibility of long (up to $130 \mathrm{ks}$ ) unbroken observations. In addition to this sample, we include $1 \mathrm{H}$ 0707-495, MS 22549-3712, NGC 4151, PHL 1092 and data from the most recent campaign of IRAS 13224-3809 (see Alston et al. 2019, 2020b for details). The total sample consists of 22 NLS1s and 16 Type-1 Seyferts, as defined by Veron-Cetty \& Veron (2010). Of particular interest are the Narrow Line Seyfert 1 AGN, which include the widely-accepted case of X-ray QPOs in RE J1034+396 (Gierlinski et al. 2008) as well as the reported case in MS 2254.9-3712 (Alston et al. 2015).

\subsection{Data Reduction}

For each AGN in our sample, we considered all observations in the XMM-Newton public HEASARC archive $^{1}$ up to September 2019 (see online table). We selected EPIC-PN data only for this analysis, as the PN has a higher throughout than the MOS detectors leading to a lower Poisson noise contribution. A circular aperture source region of 40" radius was centred on each AGN, with a background source region (also a circular aperture of 40 " radius) positioned on the same CCD, but away from the source and chip read-out direction. We then followed standard procedure of selecting only single/double pixel events (with PATTERN<=4). Using the standard XMM-Newton data reduction pipeline ${ }^{2}$, we extracted source and background light curves, using EVSELECT in SAS v17.0.0. We extracted lightcurves with a binsize of $100 \mathrm{~s}$ and utilised a sliding energy window across the nominal $0.3-10 \mathrm{keV}$ range of the instrument. This window is split into 50 approximately linear energy bins, allowing 1225 energy combinations to be studied (e.g. $0.3-0.5 \mathrm{keV}, 0.3-0.7 \mathrm{keV}$ etc.). We proceeded to use EPICLCCORR to subtract the background and apply the necessary corrections to the light curves. Whilst detector pile-up is present in some of the observations, the redistribution of

1 https://heasarc.gsfc.nasa.gov/

2 https://www.cosmos.esa.int/web/xmm-newton/sas-thread-timing counts from soft to hard energies (where the count rate is typically much lower) is not expected to lead to false QPO detections as non-QPO counts act to dilute the signal's presence. Pile-up could, however, result in QPO candidates being located in energy bands in which they are not intrinsically the strongest. We will re-visit this issue in a forthcoming paper and here re-iterate that there will be some unaccounted-for distortion.

For each observation, the high-energy $(10-12 \mathrm{keV})$, full-field background lightcurve was extracted with a binsize of 10s in order to identify soft-proton events which could contaminate the source lightcurve. We apply a cut-off such that any flares at $5 \sigma$ (where $\sigma$ is the standard deviation in the $10-12 \mathrm{keV}$ lightcurve) or more above the stable (non-flaring) count rate level are removed. This process can introduce gaps into the lightcurves, which, if left unmodified, have the potential to introduce peaks in power (making QPO detections harder). Whist there is no standard method to correct for gaps, previous approaches include linearly interpolating between short missing segments of data (e.g. < 200s), with count-rate variations accounted for by drawing from a Poisson distribution (GonzálezMartín \& Vaughan 2012; Alston et al. 2015). This method has the caveat of introducing a non-red noise background component to the light curve, which cannot replicate the component which has been removed, and thus is best applied sparingly (e.g. the interpolation used by Alston et al. 2015 is typically applied to $<0.5 \%$ of the duration of the lightcurve).

In our analysis, we take the most conservative approach of considering only the longest continuous segment between flares (see Figure 1). This avoids all interpolation assumptions, but has the limitation of reducing the amount of data available for analysis. However, we find that many of the longest continuous segments in our sample are of sufficient duration to probe down to $\leq 10^{-4} \mathrm{~Hz}$, below the frequencies of previously detected AGN QPOs (Gierlinski et al. 2008; Alston et al. 2015).

We also note that we automatically create the lightcurve of the background, the periodogram of which we can inspect to ensure that any potential QPO candidate is associated with the AGN. An example of this is shown in Figure 1, where the absence of a feature in the background confirms the QPO candidate originates in the AGN, in this case, in NGC 4051.

\section{PERIODOGRAM ANALYSIS AND QPO SEARCH}

A robust statistical framework in which to search for QPOs in broadband noise is vital (see Vaughan \& Uttley 2005), and we base our approach on that of Vaughan (2005). We produce periodograms for each energy-resolved lightcurve (reduced to $2^{n}$ bins where $n$ is an integer) by applying a fast-Fourier transform (FFT) and a fractionalrms normalisation (Vaughan et al. 2003). Using the periodogram (rather than averaged power spectral distribution: PSD) allows us to maximise the number of Fourier frequencies, improving our constraints when modelling, whilst also allowing for QPO searches down to the lowest possible frequencies in the lightcurve.

Similar to the work by González-Martín \& Vaughan (2012), we fit the periodogram using the maximum likelihood statistic. Following Vaughan (2005), we see that maximising the likelihood, $\mathcal{L}$, is equivalent to minimising $S \equiv-2 \ln [\mathcal{L}]$. Assuming a model 

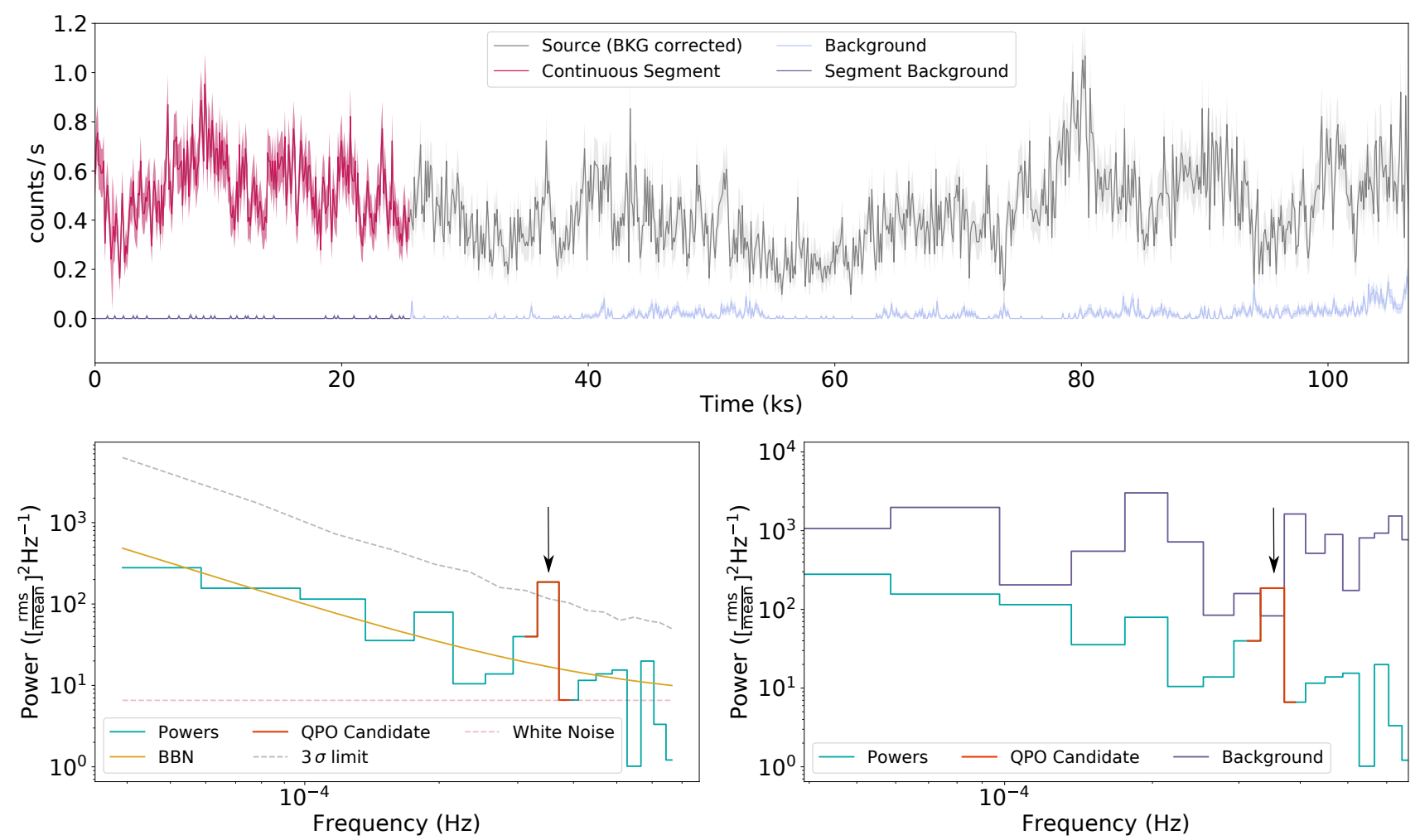

Figure 1. Top: the entire XMM-Newton EPIC-PN light curve for NGC 4051, OBS ID: 0109141401, with the longest continuous (flare-less) segment highlighted. Bottom left: periodogram of the background subtracted, flareless segment, with best fitting PLC model, simulated $3 \sigma$ line and QPO candidate highlighted. Bottom right: a direct comparison between the source and background periodogram, highlighting the lack of a corresponding peak of power in the background at the QPO candidate frequency.

$P_{j}\left(\hat{\theta}_{\mathrm{k}}\right)$, for parameters $\hat{\theta}_{\mathrm{k}}=\left(\hat{\theta}_{1} \ldots \hat{\theta}_{\mathrm{M}}\right)$, observed powers $I_{\mathrm{j}}$, and frequencies $\mathrm{j}=1,2, \ldots, n-1, S$ may be written as:

$S=2 \sum_{j=1}^{n-1}\left\{\ln \left[P_{\mathrm{j}}\right]+\frac{I_{\mathrm{j}}}{P_{\mathrm{j}}}\right\}$

We chose to apply two models to our data, the first (PLC) is a power law plus constant:

$P(v)=N_{0} v^{\beta}+C$

and consists of three free parameters: $\beta$, the spectral index, $N_{0}$, the normalisation and $C$, a constant to account for Poisson noise (white noise). The second model (BKNPLC) takes the form of a broken power-law, with break frequency, $v_{b}$ :

$P_{1}(v)=N_{1} v^{\beta_{1}}+C \quad$ for $v \leq v_{\mathrm{b}}$

$P_{2}(v)=N_{2} v^{\beta_{2}}+C \quad$ for $v>v_{\mathrm{b}}$

At frequencies at, or below the break $\left(v \leq v_{\mathrm{b}}\right)$ we assume a spectral index $\beta_{1}$ and normalisation $N_{1}$, and at frequencies above the break $\left(v>v_{\mathrm{b}}\right)$, we assume a spectral index $\beta_{2}$. The normalisation above the break, $N_{2}$, is calculated directly from the previous parameters. In both models we set the constant $(C)$ to the predicted level of the white noise following the method described in Vaughan et al. (2003):

$C=\frac{2 d t}{\langle x\rangle^{2}} \bar{\sigma}^{2}$

with timebin size, $d t$, mean count rate, $\langle x\rangle$ and mean square count rate error $\bar{\sigma}^{2}$ of the background subtracted lightcurve.

\subsection{Analysis Procedure}

We first apply the PLC model to our periodogram, using maximum likelihood statistics inbuilt into a limited memory BroydenFletcher-Goldfarb-Shanno (L-BFGS-B) minimisation algorithm, with bounds set at $(-10,10)$ for $\beta$ and $(0, \infty)$ for the normalisation, $N_{0}$. We perform a preliminary fit with this minimisation routine to obtain the parameters $\left(\hat{\beta}, \hat{N}_{0}\right)$ which are successful in minimising the negative $\log$ likelihood, $S$. Following the procedure of Vaughan (2005), we perform a frequency cut where the Poisson noise begins to dominate over the red noise (where $C>P_{\mathrm{j}}$ ), and ignore frequencies above this. We set a minimum number of Fourier frequencies in the remaining periodogram to ensure we have a sufficient number of degrees of freedom to constrain the underlying noise with simple models (in practice we have set an arbitrary lower limit of 13 Fourier bins). Note, we reject any lightcurve/energy-bin where the fitting returns a positive $\beta$, although this only occurs in the case of poor quality or Poisson noise-dominated data. 


\subsubsection{Defining the null hypothesis}

We define a QPO candidate to be a peak of power above the broadband noise, and for simplicity, that this lies within a single Fourier frequency bin. We follow Vaughan (2005), where we compute and then maximise the ratio $R=\frac{2 I_{\mathrm{j}}}{P_{\mathrm{j}}}$, comparing the observed powers $I_{\mathrm{j}}$ and the model powers, $P_{\mathrm{j}}$, across all frequencies. The frequency, $j$, in which $R_{\mathrm{j}}$ is maximised, is defined as the QPO candidate frequency, $v_{\mathrm{Q}}$, with power, $P_{\mathrm{Q}}$.

In order to determine the statistical significance of any outlier (or QPO candidate), we must first define our null hypothesis. We define our null hypothesis to be the power in the QPO candidate, $P_{\mathrm{Q}}$, being a random fluctuation of the underlying red noise, intrinsic to the AGN. Following Vaughan (2005), we remove the candidate from the periodogram (leaving $\geq 12$ bins as per our restrictions mentioned above) and refit up to the previously defined white noise cutoff frequency, with a PLC model in order to obtain the best description of the underlying continuum (the impact of fixing the position of the white noise cutoff is discussed in section 5.1). We obtain constraints on the best-fitting model parameters by applying a bootstrap-with-replacement L-BFGS-B minimisation algorithm, using maximum likelihood statistics over 10,000 iterations. This bootstrap-with-replacement method has several advantages over a single fitting iteration including reducing the significance of any individual bin variance.

For each iteration of the bootstrap, we randomly draw initial parameter estimates for use within the minimisation algorithm, using an arbitrary Gaussian distribution centred on the parameters of the initial periodogram fit $-\left(\hat{\beta}, \hat{N}_{0}\right)-$ and with a standard deviation of $\sim 10 \%$. This simple measure ensures the minimisation algorithm most effectively searches across parameter space for a true global minimum and does not prematurely meet the minimisation criteria.

Over the 10,000 bootstrap iterations, we require a minimum of $5 \%$ to fulfil the minimisation success criteria, which includes returning a negative value of $\beta$. We group the resulting distribution of successfully minimised bootstrapped parameters into 100 bins and take the $1 \sigma$ distribution about the 50th percentile, to constrain variation in mode. The modal values of these distributions represent our new minimised set of parameters $\left(\hat{\beta}, \hat{N}_{0}\right)$ which describe the PLC of the broad-band noise minus the QPO candidate.

We note that in restricting our search to excess power residing at a single Fourier frequency, any lower coherence feature which spans more than one bin will lead to us inferring a flatter powerlaw index and higher normalisation for the underlying broad band noise. We act to reduce the impact of this through our bootstrap fitting approach, which minimises the effect of individual bins on the overall model fit.

\subsubsection{Power-law vs broken power-law}

Given sufficient data quality, AGN power spectra spanning a large frequency range may be better described by a broken power law (Uttley et al. 2002; McHardy et al. 2007; González-Martín \& Vaughan 2012). To explore the presence of a break, we also fitted each periodogram with a BKNPLC, and used a Bayesian Information Criterion (BIC) test (Liddle 2007) to evaluate the statistical preference between the PLC and BKNPLC models. For the fitting process, we again used an L-BFGS-B minimisation algorithm, with bounds of (-10, $0),(-10,0),(0, \infty)$ for parameters $\beta_{1}, \beta_{2}$ and $N_{1}$ respectively, over a set of possible break frequencies: $\nu_{\mathrm{b}, \mathrm{j}}$ (we note that we require $\beta_{1}$ and $\beta_{2}$ to only take physically relevant, negative values). In order to perform a consistent BIC test, we kept the number of frequency bins the same between PLC and BKNPLC models, with possible break frequencies set to lie below the white noise cutoff frequency. Finally, we reject any maximum likelihood model fit where $\beta_{2}>\beta_{1}$. The $\mathrm{BIC}$ is defined as:

$B I C=S+k \ln (m)$

with negative log-likelihood, $S$, number of model parameters, $k$ and number of data points, $m$ (the number of data points in our periodogram). The $B I C$ value is minimised for the most statistically preferred model, i.e. it allows us to identify which model, PLC or BKNPLC, best describes our observed data (we explore the impact of noise entering from outside of our bandpass in Section 5).

Across our sample of non-white noise dominated observations (i.e. those with sufficient data points for our analysis), we find the BKNPLC model is preferred in $\sim 10 \%$ of cases. By comparison González-Martín \& Vaughan (2012), found 16\% of AGN PSDs in their sample to be best described by a bending power-law, and, as with our analysis, the majority by a simple power-law. For simplicity, we chose to focus on the remaining datasets best described with a PLC model, although we note the possibility that - should a break be present - in some cases we will lack a sufficient number of frequency bins below it to constrain its presence. We explore the impact of such unreported breaks on our QPO candidate detection and the potential impact of more complex broad band noise models - later in the paper.

\subsubsection{Uncertainty on the best-fitting PLC model}

In order to have a more complete grasp of our null hypothesis, we consider the uncertainty on the maximum likelihood parameters which describe the broad-band noise model. To do so we can follow the approach of Vaughan (2005) and use $\Delta S=-2 \Delta \ln [\mathcal{L}]$ to map confidence contours, analogous to $\Delta \chi^{2}$. For a generic Mdimensional model, we would expect the relationship between parameters to form an M-dimensional paraboloid. Vaughan (2005) demonstrates this for the simple 2-dimensional power-law case, where the relationship between parameters $\left(\beta, \log \left[N_{0}\right]\right)$ forms a highly covariant ellipse. The $1 \sigma$ parameter limits are defined following $\chi_{v}^{2}$ where in this case, $v$ is the number of model parameters (such that $1 \sigma$ for a 2-dimensional model occurs at $\Delta S=2.3$ ), and may be determined from mapping the $\Delta S$ space. Given these confidence limits, it is then possible to determine the terms of the covariance matrix (Coe 2009) describing the M-dimensional Gaussian distribution, which can then be used as the basis for Monte-Carlo simulations.

A direct approach to modelling the parameter space is to use a numerical grid; this allows the $1 \sigma$ parameter limits to be accurately determined, but is computationally intensive and also dependent on the data quality (which sets the scale of the initial grid). Semianalytical methods assuming a Gaussian symmetry in the parameter space can also provide reasonable limiting values, however, such symmetry does not hold universally. Our chosen bootstrap method (section 3.1.1) automatically constructs a discretised distribution of model parameters, centred on the best fitting values. This discrete distribution represents a true sampling of probability space, a distribution which we then seek to describe with a continuous function. To model the discretised bootstrap dataset and directly access the covariance matrix, we applied a Gaussian-mixture-model (GMM), unsupervised machine learning approach, which identifies and describes the data as a continuous model. GMM uses a single Gaussian, or sum of Gaussian distributions, with maximum likelihood statistics in its fitting process, with the covariance matrix 
defined internally. We use the GMM algorithm implemented within SCIKIT-LEARN (Pedregosa et al. 2011).

In order to determine the number of independent Gaussians needed to best describe a given boostrapped parameter space, we again apply the BIC test. For every iteration of GMM, we allow the fitting of an additional Gaussian distribution, up to a maximum of 10 iterations. The BIC penalises the added complexity of additional model parameters and the $B I C$ value is minimised in the case where the likelihood is maximised. For each iteration, the $B I C$ value is recorded, with the ideal number of GMM parameters determined from the iteration in which the $B I C$ is minimised. This method most efficiently allows us to model the $\Delta S$ parameter space with a continuous model, an example of which is shown in Figure 2.

\subsubsection{Effect of low count rates}

The combination of utilising narrow energy bands with falling count rates above the nominal response of the detector, can result in source count rates approaching the background count rate at high energies, even for the most luminous AGN (e.g. 1H 0707-495). Due to the stochastic (Poisson) nature of the background, in the case of very low source count rates, the background-subtracted lightcurve may contain bins with unphysical, negative count rates. Across our full sample of $\sim 240,000$ energy resolved lightcurves, we find a minimum of a single negative count rate bin in $\sim 30 \%$ of cases. However, as shown in Figure 3, the vast majority of such lightcurves contain only a very small fraction of negative bins overall. For those cases where negative bins are present, we find the mean proportion of negative bins to be $\sim 3 \%$ of the lightcurve, with the 90th percentile at $7 \%$.

We explore the impact of negative count rates on our analysis, by simulating red noise lightcurves (using a PLC model and the method outlined in Timmer \& Koenig (1995) and changing the fraction of negative count rates (by changing the mean count rate of the background). The bias introduced from such negative count rate bins can then be directly inferred from the difference between the input index and that determined from fitting the resulting periodogram $\left(\Delta \beta=\beta_{\text {in }}-\beta_{\text {out }}\right)$.

To perform the simulations, we utilised the best fitting models for IRAS 13224-3809, OBSID:0792180501 $\left(\beta=-1.95, \ln N_{0}=\right.$ $-5.09)$ and ARK 564 OBSID:0670130801 $\left(\beta=-0.99, \ln N_{0}=\right.$ -2.59 ) as these both have PLC models which well describe the data, with indices close to -2 and -1 respectively, allowing us to explore PLC parameter dependence. From the lightcurves generated following (Timmer \& Koenig 1995), we added Poisson noise to the lightcurves based on the corresponding mean count rate and a Poisson noise background to simulate X-ray background in the detector. To simulate a real observation, we then subtracted a new Poisson noise background, to leave the new backgroundsubtracted lightcurve, the periodogram of which we model following the method already described. We repeat the simulation 10,000 times in total (across a range of increasing background count rates) to sample the red noise, with the results shown in Figure 4. This indicates that for the vast majority of our actual lightcurves containing negative bins ( $7 \%$ at the 90th quantile level), their presence introduces a bias error on the index of $\Delta \beta<0.2$, which lies comfortably within the expected $68 \%$ confidence intervals of $\beta$ values using a PLC, as seen in Figure 4. We find this effect is slightly more pronounced in cases of steeper indices, $\beta \sim-2$, but even at the limit of the \% of negative bins observed in our real data, this effect is negligible compared to the uncertainty from fitting with a PLC model itself - as can be seen in Figure 2. We note that, for both
PLC models, the number of simulated cases which our methodology would deem to be white noise dominated (where the number of Fourier bins below the white noise cut off is $<13$ ) increases with the $\%$ of negative bins. This is especially true in simulated cases with flatter indices $(\beta \sim-1)$, where this proportion becomes very high, at $>70 \%$ of cases when considering $10 \%$ negative bins. The application of our strict cut-off has helped avoid these issues and leaves us with typically only a very small proportion of negative bins which introduce only a small source of secondary error unlikely to impact our results.

We also explore an alternative method of replacing all negative bins with zeros (as shown in Figure 4), finding that this introduces an increased level of uncertainty in the index, with $\Delta \beta$ increasing linearly with the fraction of negative bins in the time series, similarly to the case where we keep negative bins. This approach clearly does not act to reduce the impact on the measured index, $\Delta \beta$, as we introduce a non-stochastic count rate which does not reflect the nature of the stochastic source and background. We therefore gain no benefit from this method and so avoid such an approach.

\subsubsection{QPO candidate significance testing}

To evaluate the significance of outliers identified in our energyresolved periodograms, we simulated red noise power spectra, produced through the procedure detailed in Timmer \& Koenig (1995) accounting for the small white noise contribution through the addition of the constant offset, $C$, which we use as an approximation for Poisson noise in our stochastic background explicitly - as its contribution is small. For each candidate, we simulated 10,000 fake periodograms using a PLC model to describe the broad band noise, with parameters drawn at random from the mapped M-dimensional parameter space described above. This approach accounts for the intrinsic uncertainty in our estimated broad-band noise and therefore provides a more conservative significance estimate for a QPO candidate. We treated each simulated dataset in an identical manner to the real data, first by fitting each fake periodogram using a maximum likelihood minimisation routine (using a PLC model only), before identifying the largest oulier in each, removing it and refitting the underlying continuum as before. If the fitting routine was unsuccessful (as there is a low but finite chance that the fitting algorithm cannot minimise), we drew a new fake dataset, as we require the number of simulations to equal 10,000. In each successful case, we then evaluated the ratio $R_{\mathrm{TK}}=\frac{2 I_{\mathrm{TK}}}{P_{\mathrm{TK}}}$ (where, as before, $I$ indicates the observed power, and $P$ the best-fitting model power at the same frequency). A false detection occurs if we find $R_{\mathrm{TK}}>R_{\mathrm{Q}}$, at any frequency (thereby treating each frequency as an independent free trial). Across $N$ simulations, we obtain $M$ datasets with false detections and the global significance, $G S$, of the outlier via:

$G S=1-M / N$

To exceed a $3 \sigma$ significance, we require $G S>0.9973$ (equivalent to $M<27$, given $N=10,000$ ). Any outlier which meets this requirement is then considered to be a QPO candidiate - it is important to note that any such signal identified in isolation must still be treated with caution until additional evidence is obtained (e.g. the persistence of the signal across multiple observations and at similar frequencies - Alston et al. 2014 - or other characteristic behaviour which distinguishes it from the broad-band noise). In a similar fashion, we can produce $3 \sigma$ confidence limits on observed powers from our Monte-Carlo simulations following the method of Vaughan (2005) where the false alarm probability is determined 


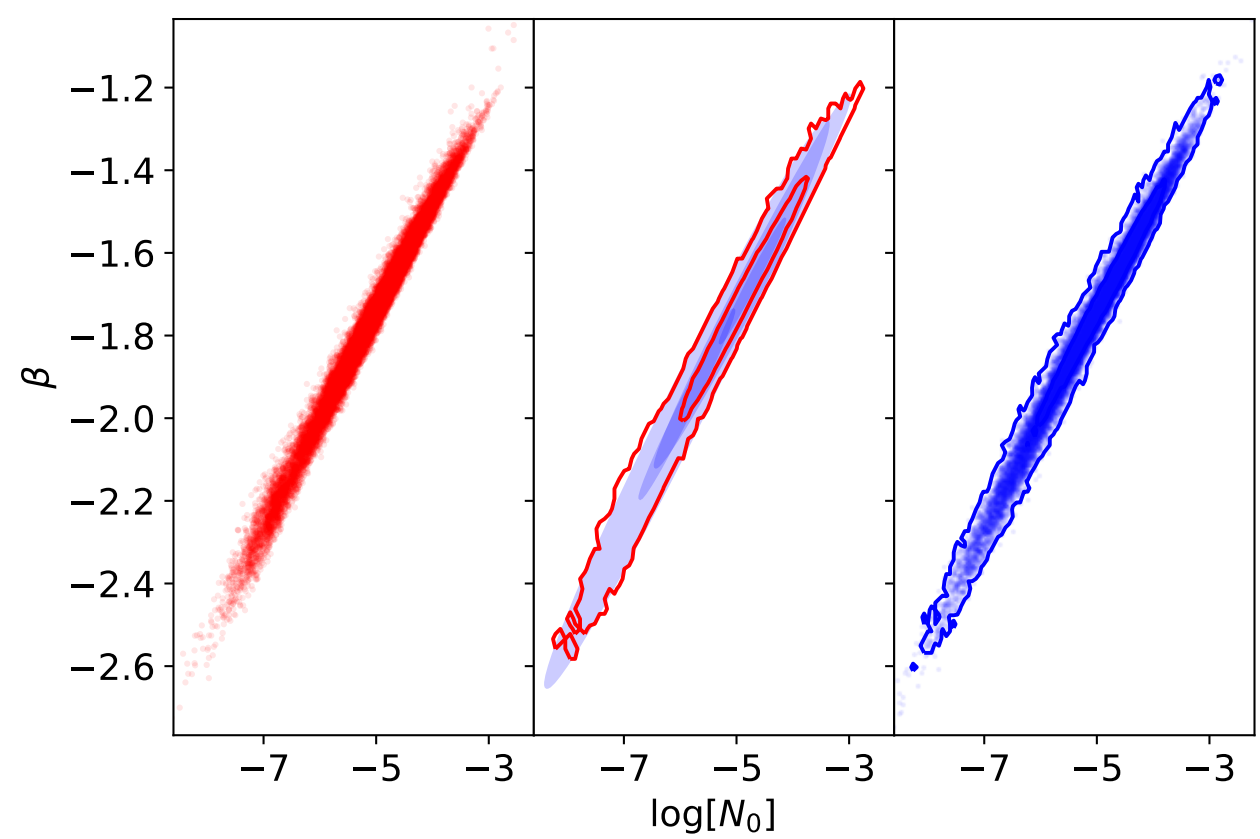

Figure 2. Plots showing our approach to modelling the parameter space of the broad-band noise, used to define our null hypothesis and constrain the QPO candidate significance. Left: discretised bootstrap distribution of PLc parameter space. Middle: a two-stage (i.e. two overlapped Gaussians) GMM model (blue), over bootstrap $\sim 68 \%$ and $\sim 99.7 \%$ probability density contours. Right: individual GMM parameter draws, with highlighted $\sim 68 \%$ and $\sim 99.7 \%$ probability density contours (i.e such that $\sim 99.7 \%$ of parameters drawn directly from the GMM lie within the outer contour).

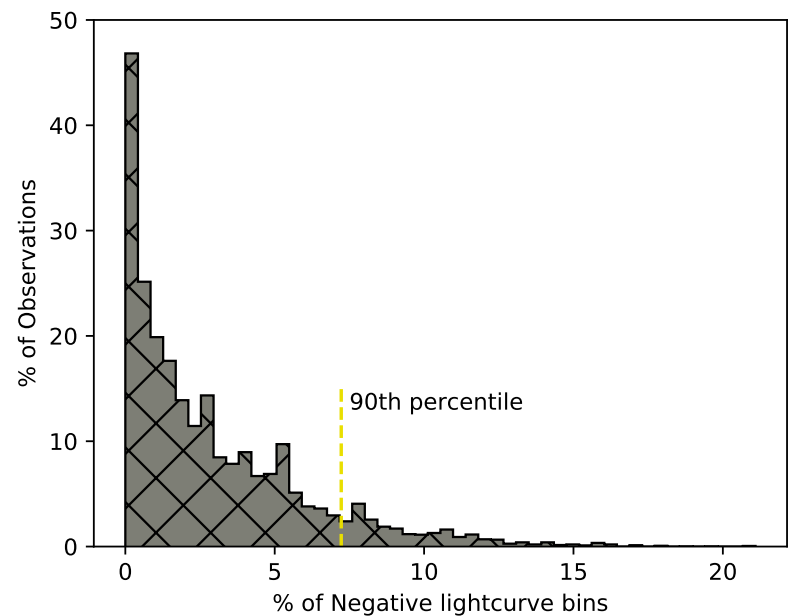

Figure 3. Histogram showing the percentage of negative count rate bins in our energy-resolved lightcurves with a minimum of a single negative bin ( $\sim 30 \%$ of sample) and with periodograms determined to be above the white noise dominated threshold ( $\geq 13$ frequency bins at frequencies below the cut-off). We find that most lightcurves have $<1 \%$ negative bins, with the 90th percentile at $\sim 7 \%$.

at the candidate frequency and then corrected post priori for the number of free trials (typically the number of frequency bins in the periodogram). Although we use the global significance described above in identifying our QPO candidates, we also display these latter $3 \sigma$ contours in our figures (e.g. Figure 1) for illustrative purposes. We also must be cautious of any detections close to the white noise cutoff, for at these frequencies, the relative fraction of Poisson noise (which we assume is constant rather than providing any additional stochastic variability) increases relative to the simulated red noise.

Whilst we treat each frequency as independent, we do not consider each energy bin to be a free trial, as we expect some level of correlation between bands (as evidenced in AGN covariance spectra - e.g. Middleton et al. 2011); we will explore techniques to address this directly in future. Finally, we note that one could take each AGN observation itself to be an independent free trial, however, as we do not yet know the conditions required to produce a QPO, nor for how long they persist, we take a more heuristic approach and treat only the frequencies as independent trials.

\subsection{Further QPO candidate significance tests}

As we have suggested earlier, it is plausible that, regardless of the fact a PLC model may be statistically preferred in a given case, our BIC test may exclude a BKNPLC model due to a low number of frequency bins below any putative break. Any unaccounted-for break in the broad-band noise has the potential to produce spurious peaks of power and so we perform a series of further tests to better judge the validity of any QPO candidates located with a PLC model.

(i) In only those energy bands where a QPO candidate is located to $\geq 3 \sigma$, we refit the entire frequency bandpass (minus the $a$ priori known QPO candidate frequency bin, but including the previously discarded white noise) with a BKNPLC model. We allow the index above and below the break $\left(\beta_{1}, \beta_{2}\right)$ and the break frequency $v_{\mathrm{b}}$ to be free (although in the case of the break set to be $\leq v_{\mathrm{Q}}$ ). We establish the new white noise cutoff frequency - which can differ from that found in the PLC fitting (see Section 5.1), and freeze the 


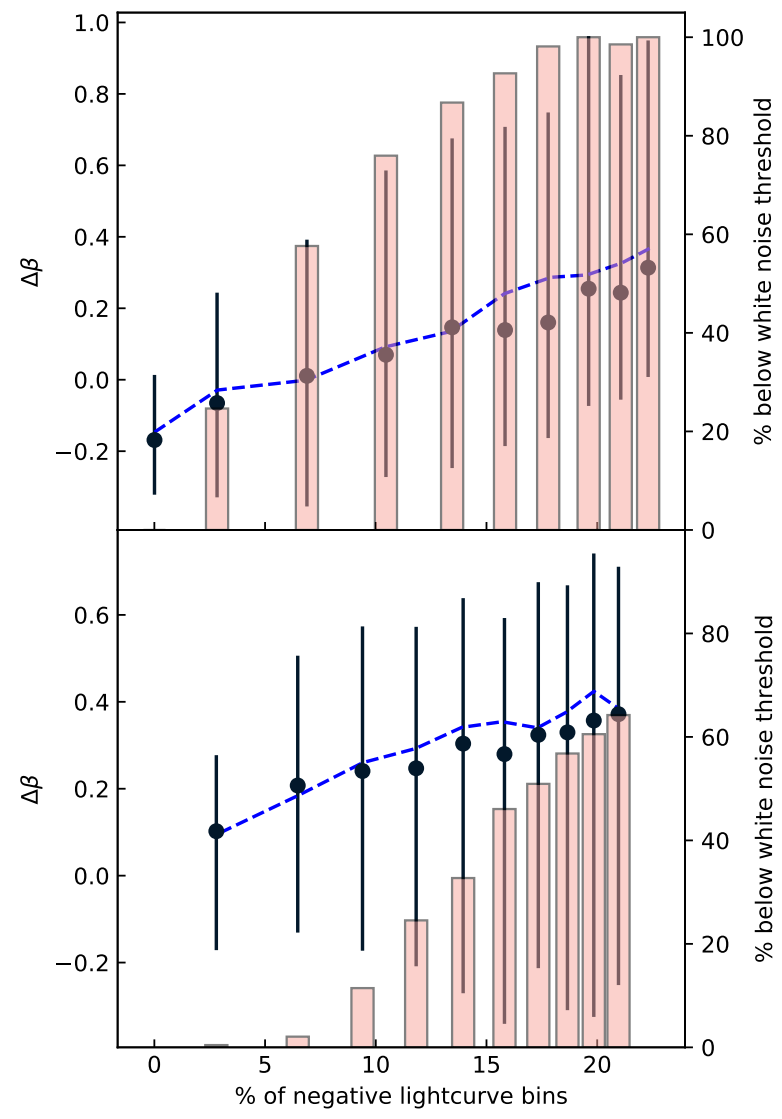

Figure 4. The impact on the inferred power spectral index, $\Delta \beta$, as a function of the percentage of negative bins found in a simulated Poisson-noise subtracted, red noise lightcurve (with $68 \%$ confidence intervals plotted). The blue dashed line shows the observed trend when we instead replace these negatives with zeros. The bars correspond to the proportion of lightcurves where the periodogram would be rejected by our requirement to have 13 or more Fourier bins at frequencies below where the white noise starts to dominate. Top panel: example case where PLC index $\beta \sim-1$, Bottom panel: example case where PLC index $\beta \sim-2$.

best fitting model (break frequency, $\beta_{1}, \beta_{2}$ and normalisation). We then simulate assuming this best-fitting model for the broad band noise and obtain a new GS value for the QPO candidate.

(ii) In only those energy bands where a QPO candidate is located to $\geq 3 \sigma$, we refit the entire frequency bandpass once again with a B KNPLC model but with a fixed lower index, $\beta_{1}$ set to -1.1 (Alston et al. 2019) and with a break frequency $v_{\mathrm{b}} \leq v_{\mathrm{Q}}$. As before, we freeze the best fitting model and obtain a new GS value for the QPO candidate.

We note that our requirement for $v_{\mathrm{b}} \leq v_{\mathrm{Q}}$ is motivated by observations of black hole binaries (notably GRS 1915+105), where the high frequency QPOs (the natural analogues to AGN QPOs at these frequencies (Middleton \& Done 2010) occur above the high frequency break in the PSD (Morgan et al. 1997).

\section{RESULTS}

From a total of 200 observations across our sample of 38 AGN, we detect 21 QPO candidates at a $\geq 3 \sigma$ significance, and 3 at a borderline $3 \sigma$ level, all from 7 AGN. We re-confirm the well-reported
QPO in 4 observations of RE J1034+396, with up to 20 additional detections across a further 6 AGN. Our initial results based on the procedure described above, are shown in Table 1 which indicate the energy band in which the detection significance is maximised, the SMBH mass and the fractional $\mathrm{rms}$ in the QPO candidate (found by integrating the QPO candidate's power and removing the Poisson noise). Although we analyse all available observations of MS 2254.9-3712, our restriction of using only continuous data prevents us from locating the QPO reported in Alston et al. (2015) as, after flare subtraction, we have a lightcurve of only $27.8 \mathrm{ks}$ in comparison to the $63 \mathrm{ks}$ combined EPIC-PN/EPIC-MOS segment defined in Alston et al. (2015) where gaps were removed via interpolation.

We note that these results are only initial, and change in significance as we perform the additional tests in the case of each AGN, as we shall proceed to describe.

\subsection{RE J1034+396}

\subsubsection{Initial PLC search}

To test our approach for locating QPO candidates, we examined the eight observations of RE J1034+396, comparing our results to those of Alston et al. (2014) noting once again that we do not include interpolation (which can restrict our frequency range).

We detect the previously reported QPO to a $>3 \sigma$ confidence, at a frequency of $2-3 \times 10^{-4} \mathrm{~Hz}$ in four observations - see Figure 5 . Notably, the maximum significance energy band extends down to $0.7 \mathrm{keV}$ and reflects the presence of the QPO in the soft X-ray component (see Middleton et al. 2011). Alston et al. (2014) detect a QPO in five of the eight observations (with the QPO now detected in new observations - Jin et al. 2020); including the four where we find a significant candidate. Whilst we do not detect a QPO in OBSID:0655310101, we select only the first $28.2 \mathrm{ks}$ as a flare-less segment, and subsequently detect a QPO at only a $>2 \sigma$ level.

In Figure 6, we present energy-resolved significance heat-maps which indicate the $\geq 3 \sigma$ significant detections detailed in Table 1 , as well as the global significance of any signal at this specific frequency in all the other energy bands. We note that the significances we present in Figure 6 are purely blind (i.e. the full number of independent trials is still taken into account).

\subsubsection{Further tests}

As the QPO in RE J1034+396 is well established and thoroughly investigated in the literature (e.g. Vaughan 2010), we refrain from applying our additional tests in this instance.

Frequency-restricted search: Given our detection of multiple $\geq 3 \sigma$ QPO candidates at consistent frequencies, we make the reasonable assumption that we have a-priori knowledge of the QPO frequency in RE J1034+396, across any observation. We then test whether a signal is located at this frequency - in this case the modal frequency of $2.34 \times 10^{-4} \mathrm{~Hz}$ (or the closest Fourier frequency) - in those observations without blind detections. In effect, this separate p-value test lowers the effective threshold for detection, as it reduces the number of free trials to a single Fourier bin of interest. However, this further analysis does not locate any additional QPO candidates at $\geq 3 \sigma$. 
Table 1. $3 \sigma$ QPO candidate summary. The columns indicate: (1) Observation ID, (2): The longest continuous segment duration of the light curve, (3): the highest value of global significance obtained in a single energy bin, (4): the energy range of this bin, (5): the Fourier frequency of the QPO candidate detected at $\geq 3 \sigma,(6)$ : the Fourier frequency bin width, (7): the (peak significance) fractional rms (FRMS) value at the QPO candidate Fourier frequency, (8): the QPO candidate Quality factor $\left(Q=\frac{v}{\Delta v}\right.$ which we point out is not the product of the duration and QPO candidate frequency due to restricting our lightcurve to $2^{n}$ bins). * We note that IRAS 13224-3809, OBSID:0792180201, 1H 0707-495 OBSID: 0653510601 and MRK 766, OBSID:0304030301 are considered borderline $3 \sigma$ results with ambiguous detections assuming a PLC model. $\dagger$ highlighted observations are those which are not validated by our further tests (see Section 3.2). All SMBH masses are provided in the form: $\log _{10}\left(\frac{M}{M_{\odot}}\right)$ The estimated SMBH masses are obtained from: ${ }^{b}$ Done et al. (2012a), ${ }^{c}$ Alston et al. (2019), ${ }^{d}$ Done \& Jin (2016), ${ }^{e}$ Gierliński \& Done (2004), ${ }^{f}$ Denney et al. (2010), ${ }^{g}$ Wang, T. \& Lu, Y. (2001), ${ }^{h}$ Giacché et al. (2014).

\begin{tabular}{|c|c|c|c|c|c|c|c|}
\hline Obs ID & Duration (ks) & $\begin{array}{c}\text { Peak } \\
\text { Significance (GS) }\end{array}$ & $\begin{array}{c}\text { Peak } \\
\text { Energy }(\mathrm{keV})\end{array}$ & $\begin{array}{l}\text { QPO candidate } \\
\text { Frequency }(\mathrm{Hz})\end{array}$ & $\begin{array}{l}\text { Frequency } \\
\text { Error }(\mathrm{Hz})\end{array}$ & $\begin{array}{l}\text { FRMS } \\
\text { Value }\end{array}$ & $\mathrm{Q}$ \\
\hline \multicolumn{8}{|c|}{ RE J1034+396, $M_{\mathrm{BH}} \sim 6.17^{a b}$} \\
\hline 0506440101 & 28.94 & 1.0000 & $0.7-1.0$ & $2.34 \times 10^{-4}$ & $3.91 \times 10^{-5}$ & 0.071 & 5.98 \\
\hline 0655310201 & 41.60 & 1.0000 & $1.2-2.4$ & $2.34 \times 10^{-4}$ & $3.91 \times 10^{-5}$ & 0.123 & 5.98 \\
\hline 0675440101 & 23.92 & 1.0000 & $0.7-3.4$ & $2.34 \times 10^{-4}$ & $7.81 \times 10^{-5}$ & 0.097 & 3.00 \\
\hline 0675440201 & 28.82 & 1.0000 & $0.9-6.2$ & $2.73 \times 10^{-4}$ & $3.91 \times 10^{-5}$ & 0.112 & 5.98 \\
\hline \multicolumn{8}{|c|}{ IRAS 13224-3809, $M_{\mathrm{BH}} \sim 6.00^{c}$} \\
\hline$\dagger 0780561301$ & 125.29 & 0.9991 & $6.0-10.0$ & $7.81 \times 10^{-5}$ & $9.77 \times 10^{-6}$ & 0.201 & 8.00 \\
\hline 0780561301 & 125.29 & 0.9980 & $2.2-6.4$ & $2.83 \times 10^{-4}$ & $9.77 \times 10^{-6}$ & 0.121 & 29.0 \\
\hline$\dagger 0780561401$ & 82.92 & 1.0000 & $4.0-5.8$ & $7.81 \times 10^{-5}$ & $1.95 \times 10^{-5}$ & 0.279 & 4.00 \\
\hline 0780561401 & 82.92 & 0.9997 & $3.2-9.8$ & $4.69 \times 10^{-4}$ & $1.95 \times 10^{-5}$ & 0.136 & 24.1 \\
\hline 0780561701 & 113.20 & 0.9995 & $3.2-3.8$ & $9.77 \times 10^{-5}$ & $9.77 \times 10^{-6}$ & 0.209 & 10.0 \\
\hline * 0792180201 & 128.63 & 0.9979 & $4.0-5.6$ & $1.66 \times 10^{-4}$ & $9.77 \times 10^{-6}$ & 0.294 & 17.0 \\
\hline$\dagger 0792180301$ & 53.26 & 0.9994 & $4.8-9.6$ & $9.77 \times 10^{-5}$ & $1.95 \times 10^{-5}$ & 0.304 & 5.01 \\
\hline 0792180501 & 93.83 & 0.9992 & $0.9-1.6$ & $1.02 \times 10^{-3}$ & $1.95 \times 10^{-5}$ & 0.059 & 52.3 \\
\hline 0792180601 & 105.02 & 0.9984 & $6.6-8.6$ & $9.77 \times 10^{-5}$ & $9.77 \times 10^{-6}$ & 0.246 & 10.0 \\
\hline \multicolumn{8}{|c|}{$1 \mathrm{H} 0707-495, M_{\mathrm{BH}} \sim 6.3^{c}$} \\
\hline 0653510301 & 95.19 & 0.9984 & $4.8-6.2$ & $1.95 \times 10^{-4}$ & $1.95 \times 10^{-5}$ & 0.255 & 10.0 \\
\hline 0653510501 & 94.72 & 0.9997 & $4.0-5.4$ & $1.95 \times 10^{-4}$ & $1.95 \times 10^{-5}$ & 0.225 & 10.0 \\
\hline * 0653510601 & 105.85 & 0.9975 & $5.6-8.2$ & $1.27 \times 10^{-4}$ & $9.77 \times 10^{-6}$ & 0.224 & 13.0 \\
\hline \multicolumn{8}{|c|}{ PG1244+026, $M_{\mathrm{BH}} \sim 6.24^{d}$} \\
\hline 0675320101 & 71.81 & 0.9993 & $3.0-5.8$ & $2.15 \times 10^{-4}$ & $1.95 \times 10^{-5}$ & 0.074 & 11.0 \\
\hline \multicolumn{8}{|c|}{ NGC 4051, $M_{\mathrm{BH}} \sim 6.24^{e}$} \\
\hline 0109141401 & 25.77 & 0.9997 & $5.0-6.0$ & $3.52 \times 10^{-4}$ & $3.91 \times 10^{-5}$ & 0.095 & 9.00 \\
\hline$\dagger 0606321801$ & 39.83 & 0.9998 & $3.4-4.0$ & $1.56 \times 10^{-4}$ & $3.91 \times 10^{-5}$ & 0.180 & 3.99 \\
\hline \multicolumn{8}{|c|}{ ARK 564, $M_{\mathrm{BH}} \sim 6.41^{f}$} \\
\hline$\dagger 0006810101$ & 4.95 & 1.0 & $3.0-5.4$ & $1.88 \times 10^{-3}$ & $3.13 \times 10^{-4}$ & 0.134 & 6.00 \\
\hline 0670130501 & 17.18 & 0.9987 & $2.0-3.8$ & $8.59 \times 10^{-4}$ & $7.81 \times 10^{-5}$ & 0.073 & 11.0 \\
\hline 0670130701 & 44.71 & 1.0 & $2.6-2.8$ & $2.73 \times 10^{-4}$ & $3.91 \times 10^{-5}$ & 0.163 & 6.98 \\
\hline 0670130801 & 51.65 & 0.9998 & $3.0-3.4$ & $2.54 \times 10^{-4}$ & $1.95 \times 10^{-5}$ & 0.072 & 13.0 \\
\hline \multicolumn{8}{|c|}{ MRK 766, $M_{\mathrm{BH}} \sim 6.63^{g}$} \\
\hline$* \dagger 0304030301$ & 38.35 & 0.9976 & $3.0-4.6$ & $4.69 \times 10^{-4}$ & $3.91 \times 10^{-5}$ & 0.055 & 12.0 \\
\hline
\end{tabular}

\subsection{IRAS 13224-3809}

IRAS 13224-3809 is one of the most variable NLS1 AGN and has been the focus of a recent $1.5 \mathrm{Ms}$ study with XMM Newton, yielding high quality data with almost full orbits (see Alston et al. 2019, Alston et al. 2020b). We studied 16 available observations, and in 6 of these detect QPO candidates at $\geq 3 \sigma$, with one additional observation with a borderline $3 \sigma$ detection.

\subsubsection{Initial PLC search}

Three observations all have consistent QPO candidate frequencies at $9.77 \times 10^{-5} \mathrm{~Hz}$ (OBSIDs:0780561701, 0792180301 and 0792180601) with an extremely high fractional rms (> 20\%) see Figure 7 In addition to these, we find a borderline $3 \sigma$ QPO candidate with a frequency of $1.66 \times 10^{-4} \mathrm{~Hz}$ in OBSID:0792180201; although the significance fluctuates around $3 \sigma$, it sits close to the frequency of QPO candidates detected in other observations and is therefore of interest.

We also detect a $>3 \sigma$ QPO candidate at $7.81 \times 10^{-5} \mathrm{~Hz}$ in two observations (OBSID:0780561301 and 0780561401). These two observations provide a potentially intriguing result in that they contain $>3 \sigma$ QPO candidates at $t w o$ different frequencies, dependent on the energy band selected, both of which are presented in Table 1. In OBSID:0780561301, the higher frequency QPO candidate is found at $2.83 \times 10^{-4} \mathrm{~Hz}$, whilst in OBSID:0780561401 it is found at $4.69 \times 10^{-4} \mathrm{~Hz}$. If we assume these to be harmonically related to the $7.81 \times 10^{-5} \mathrm{~Hz}$ feature then these would be at $7: 2$ and $6: 1$ resonances respectively. Figure 8 displays the energy dependence of the global significance of these QPO candidates; the energies at which they become statistically significant at $\geq 3 \sigma$, are almost entirely confined to hard energies $>3 \mathrm{keV}$. The notable exception to the energy-dependence is that of OBSID:0792180501, which appears to show several features that are unique amongst our sample.

The QPO candidate in OBSID:0792180501 is found at a still higher frequency of $1.02 \times 10^{-3} \mathrm{~Hz}$, as shown in Figure 9. This QPO candidate is almost unique amongst our detections (the other example being found in ARK 564), as it lies at a frequency higher than the Poisson noise cutoff for the vast majority of our observations, and we only detect it due to the high count rates. In combination 


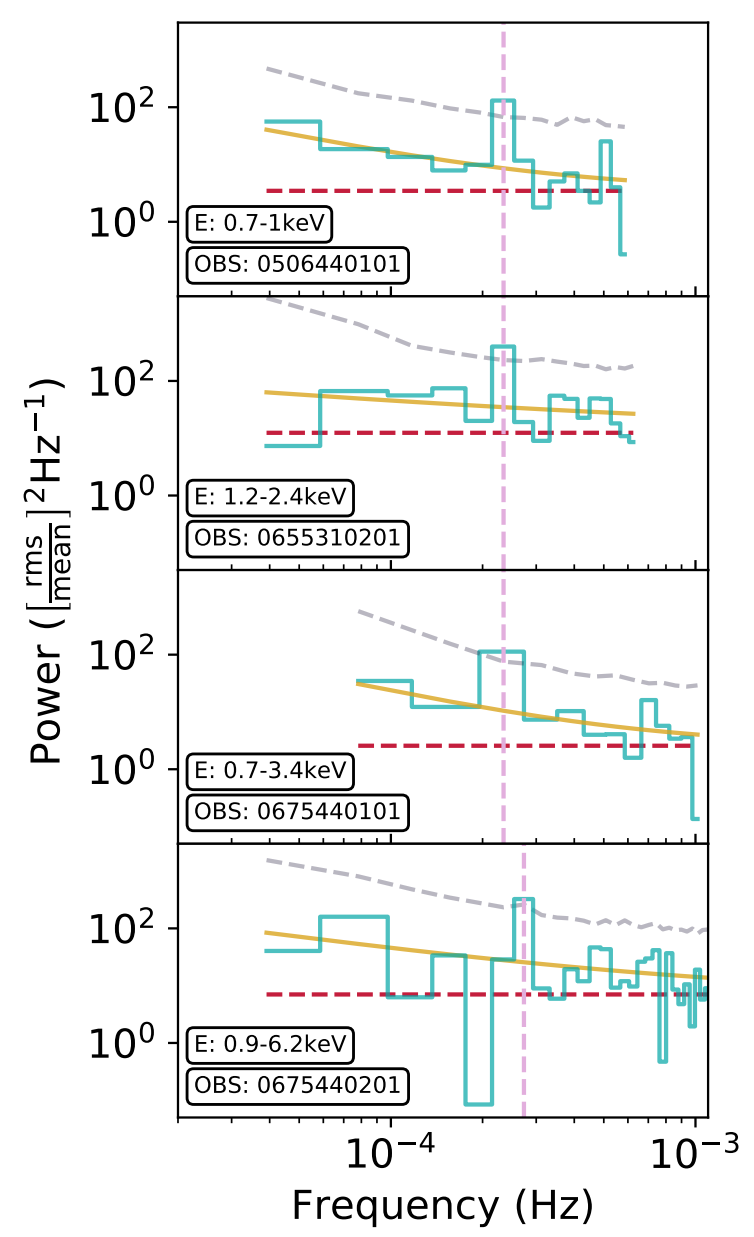

Figure 5. The highest significance $(\geq 3 \sigma)$ QPO candidates detected in RE J1034+396, using the PLC model, with the corresponding frequency bin highlighted. The consistency of the QPO frequency across each observation has historically set RE J1034+396 apart as it is highly improbable to create false peaks in power across multiple observations at the same frequency.

with the length of this observation (setting the size of the natural Fourier frequency bin), this QPO candidate has the largest $Q$ value of any of our detections $(Q>50)$. The energy band at which the significance of this QPO candidate is maximised also differs from those of the lower frequency QPO candidates, being found at softer energies (specifically $0.9-1.6 \mathrm{keV}$ - see Figure 8) without significant detections above $2 \mathrm{keV}$ (although at these higher energies, the data quality becomes insufficient to study such high frequencies). It is of interest to note that those QPO candidates detected at lower frequencies do not appear in this observation.

\subsubsection{Further tests}

We proceed to apply the two additional tests discussed in Section 3.2 and describe the results on an observation-by-observation basis.

Test(i):

- OBSID:0780561301. We find a break at $\sim 2 \times 10^{-4} \mathrm{~Hz}$ at energies $\sim 2-6 \mathrm{keV}$. In this case we observe a sudden drop in power above the high frequency QPO candidate at $2.83 \times 10^{-4} \mathrm{~Hz}$, as shown in the lower panel of Figure 10. This behaviour may not be best described by a break but is similar to the findings of González-Martín \& Vaughan (2012) when modelling the PSD of MS 22549-3712, in which they report a poorly constrained fit using a bending-power law with an extremely steep index above the break, but no QPO. Assuming a free BKNPLC model does not lower the significance of the higher frequency candidate QPO in this observation. In the case of energies $>6 \mathrm{keV}$, we find a break at $\sim 2 \times 10^{-5} \mathrm{~Hz}$ or $\sim 6 \times 10^{-5} \mathrm{~Hz}$, and in doing so, the significance of the QPO candidate at $7.81 \times 10^{-5} \mathrm{~Hz}$ drops to below $3 \sigma-$ as shown in upper panel of Figure 10. By including a break, the white noise cutoff (i.e. the frequency at which the Poisson noise dominates over the red noise) is brought to lower frequencies and restricts the number of frequency bins in the periodogram; as a result, we find multiple energy bands which contained $\geq 3 \sigma$ QPO candidates using the PLC model, to now be white noise dominated, i.e. with $<13$ Fourier bins below the cutoff (which technically includes the QPO candidate bin, although the power in this bin is ignored in defining the null hypothesis).

- OBSID:0780561401. We find the high-frequency QPO candidate at $4.69 \times 10^{-4} \mathrm{~Hz}$ lies consistently above the $3 \sigma$ threshold when within the white noise limit, see upper panel of Figure 11. For energy ranges above $3.2 \mathrm{keV}$ however, we find the white noise cutoff moves to frequencies below $4.69 \times 10^{-4} \mathrm{~Hz}$ and the QPO candidate is no longer considered. We also find that the low-frequency QPO candidate at $7.81 \times 10^{-5} \mathrm{~Hz}$ may be well described by a break across an energy range of $2-10 \mathrm{keV}$, although the strength of the QPO candidate increases with increasing energies and becomes less well described by a break given our methodology, see lower panel of Figure 11. Given the result of this test, we remain cautious of the validity of this low-frequency QPO candidate and believe it requires further investigation.

- OBSID:0780561701. We find a weak break (i.e. a value of $\beta_{1} / \beta_{2}$ close to unity) at $\sim 2 \times 10^{-5} \mathrm{~Hz}$ and at energies $>3 \mathrm{keV}$, which has no impact on the significance of the QPO candidate at $9.77 \times 10^{-5} \mathrm{~Hz}$.

- OBSID:0792180201. We reported only a borderline detection using a PLC model. We subsequently find a break at $\sim 8 \times 10^{-5}$ $\mathrm{Hz}$, however, the QPO candidate at $1.66 \times 10^{-4} \mathrm{~Hz}$ becomes more significant.

- OBSID:0792180301. We lose the QPO candidate at $9.77 \times$ $10^{-5} \mathrm{~Hz}$ entirely with a weak break at $\sim 4 \times 10^{-5} \mathrm{~Hz}$ and before a steep drop in power at frequencies above the QPO candidate. We find that neither the PLC or BKNPLC models provide a good description of the broad band noise, but in assuming a BKNPLC model, the white noise cutoff is brought to sufficiently low frequencies for the data to be white noise dominated.

- OBSID:0792180501. We find a weak break around $\sim 4 \times 10^{-5}$ $\mathrm{Hz}$, but the QPO candidate at $1.02 \times 10^{-3} \mathrm{~Hz}$ remains significant.

- OBSID:0792180601. The lower frequency white noise cutoff means we are close to datasets becoming white noise dominated, however, the QPO candidate at $9.77 \times 10^{-5} \mathrm{~Hz}$ remains significant on the edge of our frequency range.

\section{Test(ii):}

All of the results with a fixed $\beta_{1}$ agree with our findings from test(i), (as expected given $\beta_{1}=-1.1$ was found previously in the case of IRAS 13224-3809 Alston et al. 2019) although this assumed shape for the broad band noise does not always provide a good description depending on the energy range under consideration.

The QPO candidates identified through our initial search using 

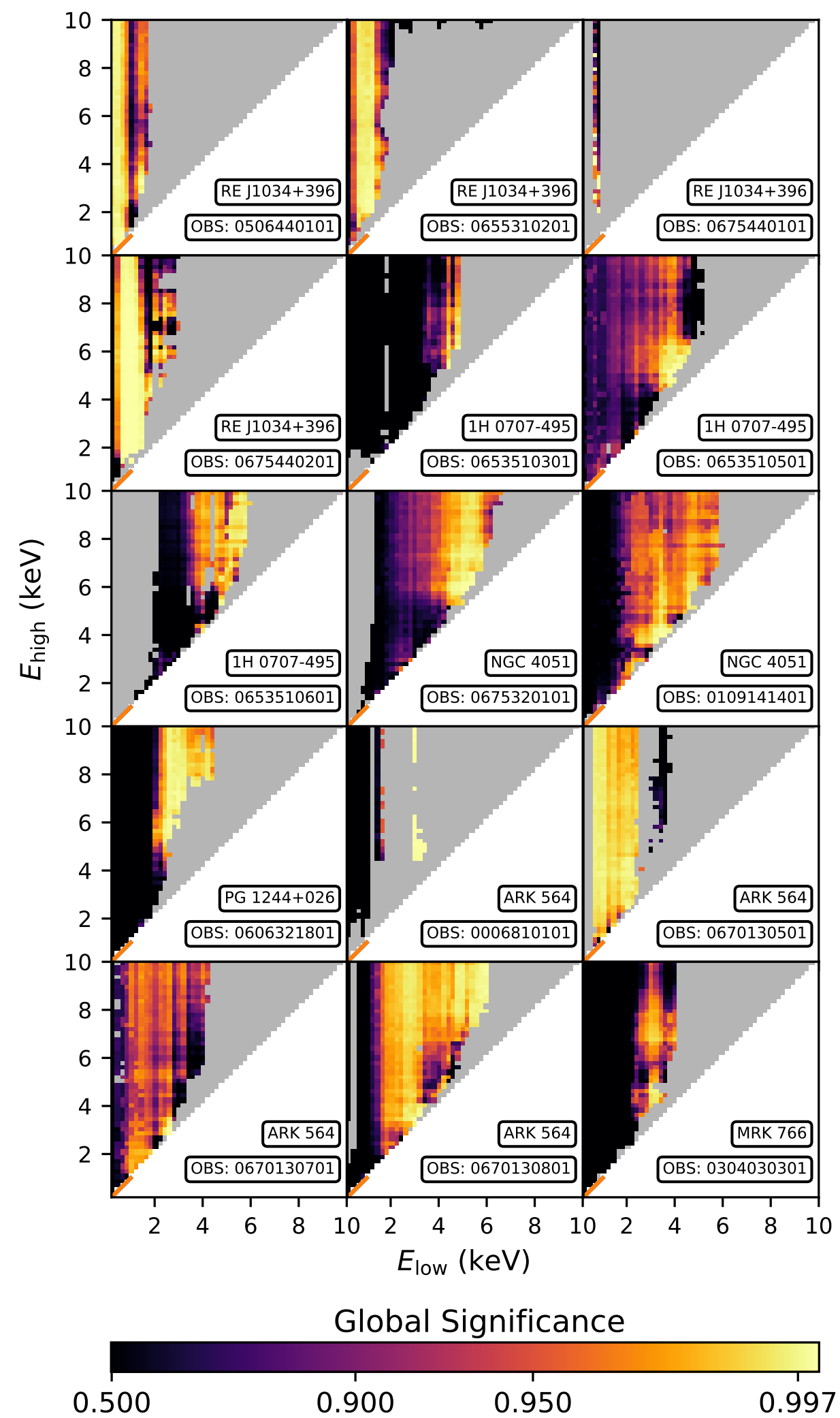

Figure 6. Energy-resolved significance heatmaps displaying the measured global significance of QPO candidates (excluding IRAS 13224-3809, for which see Figure 8) using the PLC model, at the frequency of the highest significance blind detection in an energy bin defined as $\mathrm{E}_{\text {low }} \rightarrow \mathrm{E}_{\mathrm{high}}$ The grey areas, including 'gaps' in the heatmaps, indicate energy bands which do not fit the PLC criteria we set out in Section 3 - including both white noise dominated observations and BKNPLC model preferred fits. 


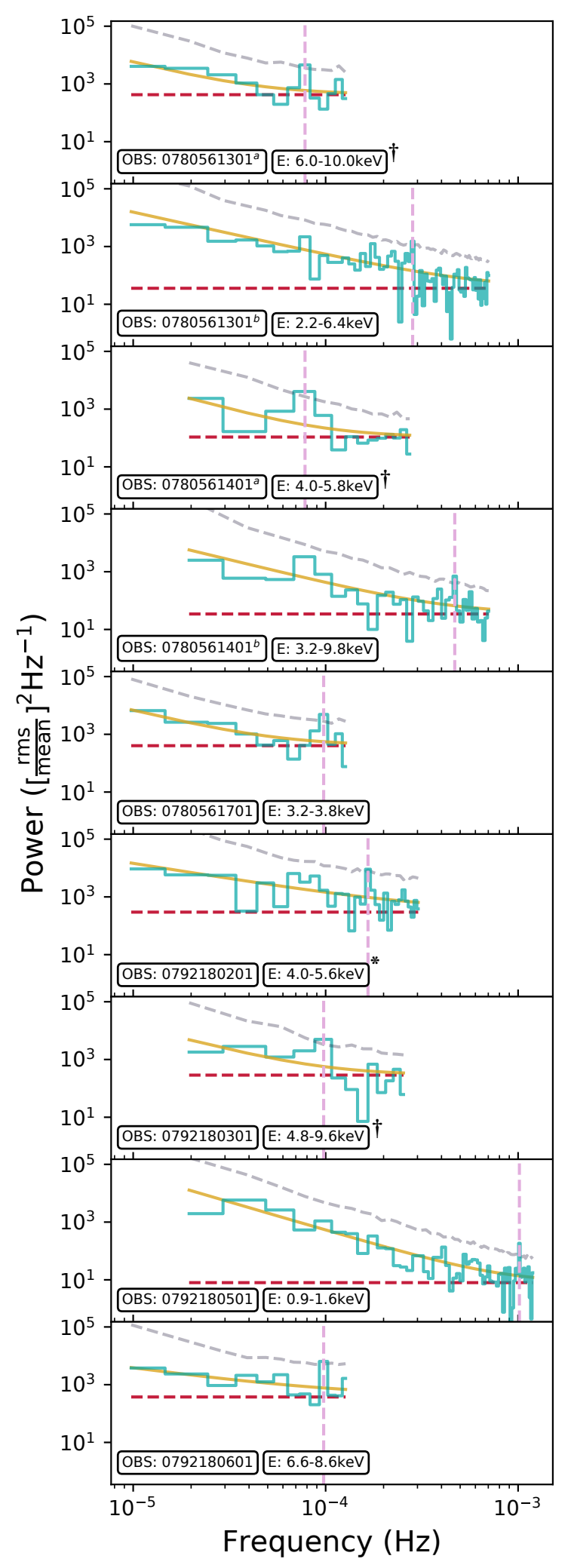

Figure 7. The highest significance $(\geq 3 \sigma)$ QPO candidates found in IRAS 13224-3809 using the PLC model, with the corresponding frequency bin highlighted. We note the consistency of frequencies across multiple observations, with the clear exception of OBSID:0792180501. See Table 1 for supplementary information. We highlight borderline $3 \sigma$ results $(*)$ and those which are not validated by our further tests $(\dagger-$ see Section 3.2). the PLC model, but where the above tests question their validity, are highlighted in Table 1 and the respective figures.

Frequency-restricted search: As with RE J1034+396, the detection of multiple $\geq 3 \sigma$ QPO candidates at consistent frequencies, allows us to assume a-priori knowledge of a possible QPO candidate at frequencies of $7.81 \times 10^{-5} \mathrm{~Hz}$ and $9.77 \times 10^{-5} \mathrm{~Hz}$ (or the closest Fourier frequency). Assuming a frequency of $9.77 \times 10^{-5} \mathrm{~Hz}$, we find a frequency-restricted $\geq 3 \sigma$ QPO candidate at energy ranges $>3 \mathrm{keV}$ in OBSID:0673580401. We note that this observation does not show any $\geq 3 \sigma$ global significance features, so does not appear in Table 1. Assuming a frequency of $7.81 \times 10^{-5} \mathrm{~Hz}$, we find a single frequency-restricted $\geq 3 \sigma \mathrm{QPO}$ candidate at $2.8-3 \mathrm{keV}$ in OBSID:0792180201.

In light of the possible harmonic features observed in OBSIDs:0780561301 and 0780561401, we also search for QPO candidates at frequencies of $2.83 \times 10^{-4} \mathrm{~Hz}$ and $4.69 \times 10^{-4} \mathrm{~Hz}$ respectively. At a frequency of $2.83 \times 10^{-4} \mathrm{~Hz}$, we find frequency-restricted $\geq 3 \sigma$ candidates in OBSIDs: 0673580101 and 0780561601 , both in the 3 - $6 \mathrm{keV}$ range, comparable to the QPO candidate in OBSID:0780561301. At a frequency of $4.69 \times 10^{-4} \mathrm{~Hz}$, we also find frequency-restricted $\geq 3 \sigma$ candidates in OBSID:0673580101 in the $2-8 \mathrm{keV}$ range, and in OBSID:0780560101, at energies around $2 \mathrm{keV}$. The apparent detection of a higher frequency QPO candidate in the same observation (OBSID:0673580101) but at both frequencies highlights the potential problem with searching at a reduced number of frequencies: even with an a priori expectation - the significance is artificially higher and care must be taken when claiming any detection (i.e. independent evidence is still required).

\subsection{H 0707-495}

1H 0707-495 has been studied extensively by XMM-Newton with a total observing time in excess of 1 Ms (e.g. Kara et al. 2013).

\subsubsection{Initial PLC search}

As shown in Figure 12, we detect $\geq 3 \sigma$ QPO candidates in three observations (including one borderline detection), from a total of 11 , with high fractional $\mathrm{rms}(>20 \%)$ and across a narrow frequency range of $1-2 \times 10^{-4} \mathrm{~Hz}$.

We find a borderline $\sim 3 \sigma$ QPO candidate in OBSID:0653510601, although in this case, the QPO candidate frequency lies close to or even at the white noise cutoff in some energy bins. For this observation, we find a slight shift towards lower frequencies, but there is minimal change in the fractional-rms of the QPO candidate between each of these observations.

We find the peak QPO candidate significance in OBSID:0653510301 and 0653510501 to lie in the $\sim 4-6 \mathrm{keV}$ range, with no $\geq 3 \sigma$ energy bands extending below $3.8 \mathrm{keV}$, whilst we find only borderline $3 \sigma$ features in OBSID:0653510601 in the $\sim 4$ - $8 \mathrm{keV}$ energy range. The extent of the significance variation is highlighted in Figure 6. We note also that the fractional-rms of the QPO candidates in $1 \mathrm{H} 0707-495$ is similar to that found in IRAS 13224-3809.

\subsubsection{Further tests}

Test(i): 


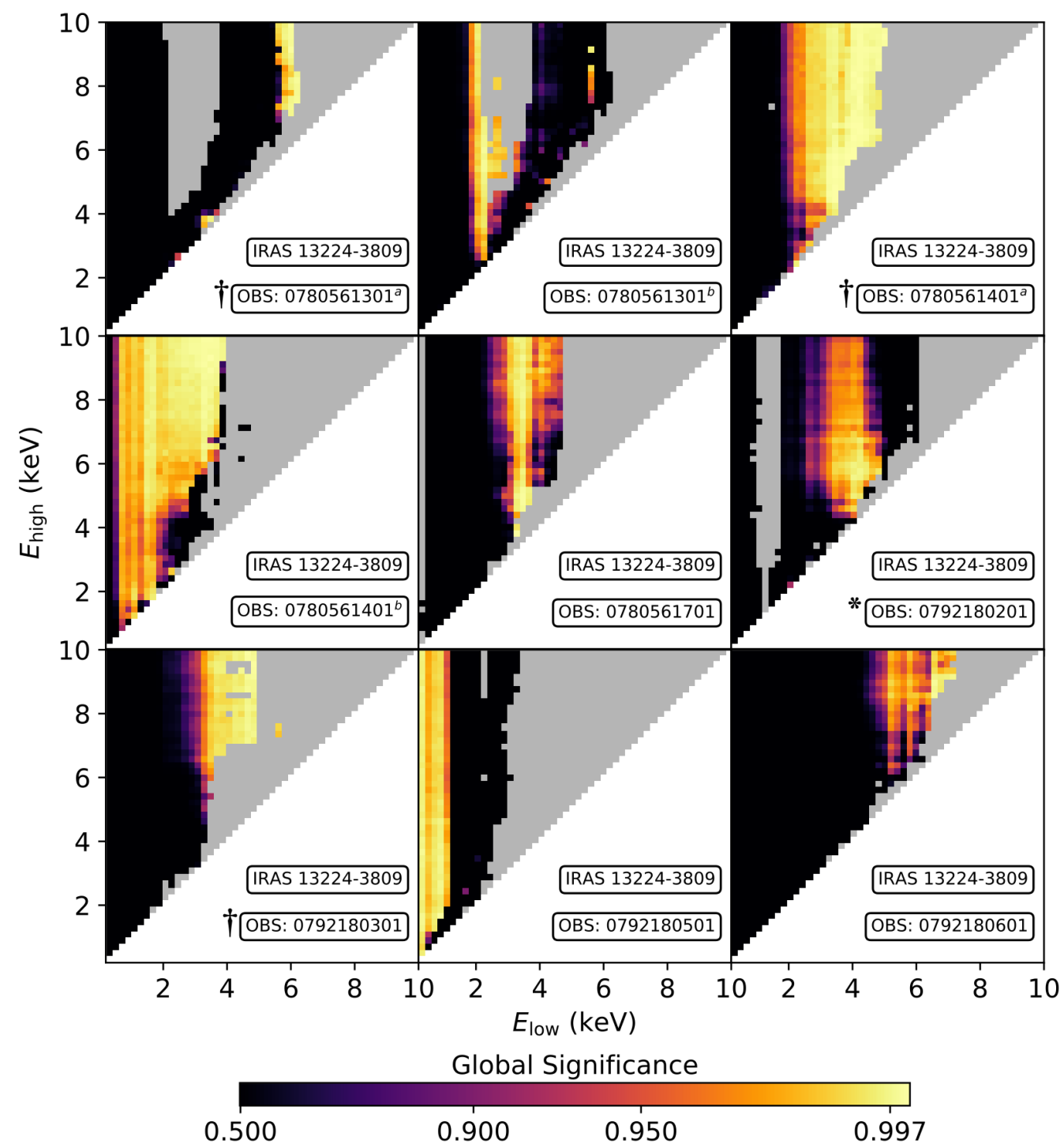

Figure 8. Energy-resolved significance heatmaps displaying the measured global significance of the QPO candidates in IRAS 13224-3809, using the PLC

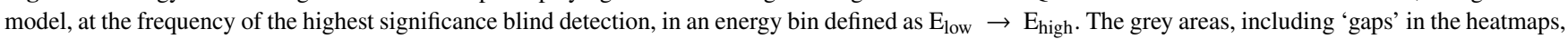
indicate energy bands which do not fit the PLC criteria we set out in Section 3 - including both white noise dominated observations and BKNPLC model preferred fits.

- OBSID:0653510301. Assuming a free BKNPLC model, we find a weak break at $\sim 1 \times 10^{-4} \mathrm{~Hz}$. The biggest impact is the shift of the white noise cutoff; in the $4.4-5.4 \mathrm{keV}$ energy range the QPO candidate remains above the $3 \sigma$ threshold but at the limit of non white noise dominated bins. As we move to harder energy bands, (namely $4.8-6.2 \mathrm{keV}$ ), the QPO candidate sits at the location of the white noise cutoff and we thus cannot discern its significance.

- OBSID:0653510501. In the $3.8-5.4 \mathrm{keV}$ band, the periodogram is best described by a break at $\sim 8 \times 10^{-5} \mathrm{~Hz}$, whilst at $4.2-5.8 \mathrm{keV}$, the break extends back to $\sim 3 \times 10^{-5} \mathrm{~Hz}$. In both cases, the QPO candidate at $1.95 \times 10^{-4} \mathrm{~Hz}$ remains $\geq 3 \sigma$. As with the previous observation, there are also energy bands in which we are now restricted by the white noise cutoff. This is seen in narrower energy bands (e.g. $3.8-4.8 \mathrm{keV}$ and $4.2-5.4 \mathrm{keV}$ ), as the number of counts is restricted. In these cases, the QPO candidate remains $\geq 3 \sigma$ but at a frequency closer to the white noise cutoff.

- OBSID:0653510601. A borderline detection with a PLC model, when assuming a BKNPLC model we find a tentative break around the QPO candidate frequency $\sim 1 \times 10^{-4} \mathrm{~Hz}$. For this observation, we are investigating only two $\geq 3 \sigma$ energy bands, where the QPO candidate is found at frequencies below the PLC white noise cutoff. For the peak significance band $(5.6-8.2 \mathrm{keV})$, we find the QPO candidate remains $\geq 3 \sigma$, whilst in the $4.8-5.4 \mathrm{keV}$ band, the QPO candidate falls below our detection threshold due to the presence of the break.

\section{Test(ii):}

- OBSID:0653510301. Similar to the free-index case above, we find the QPO candidate remains significant above the broad band noise, but with a white noise cutoff located at a lower frequency which reduces the number of frequency bins below our limit.

- OBSID:0653510501. Due to the forced upper index $\beta_{1}=-1.1$ providing a poor description of the power spectrum of $1 \mathrm{H} 0707-495$, we find only a limited number of breaks with this model. Notably, we find that the $\geq 3 \sigma$ detection remains in the peak significance 


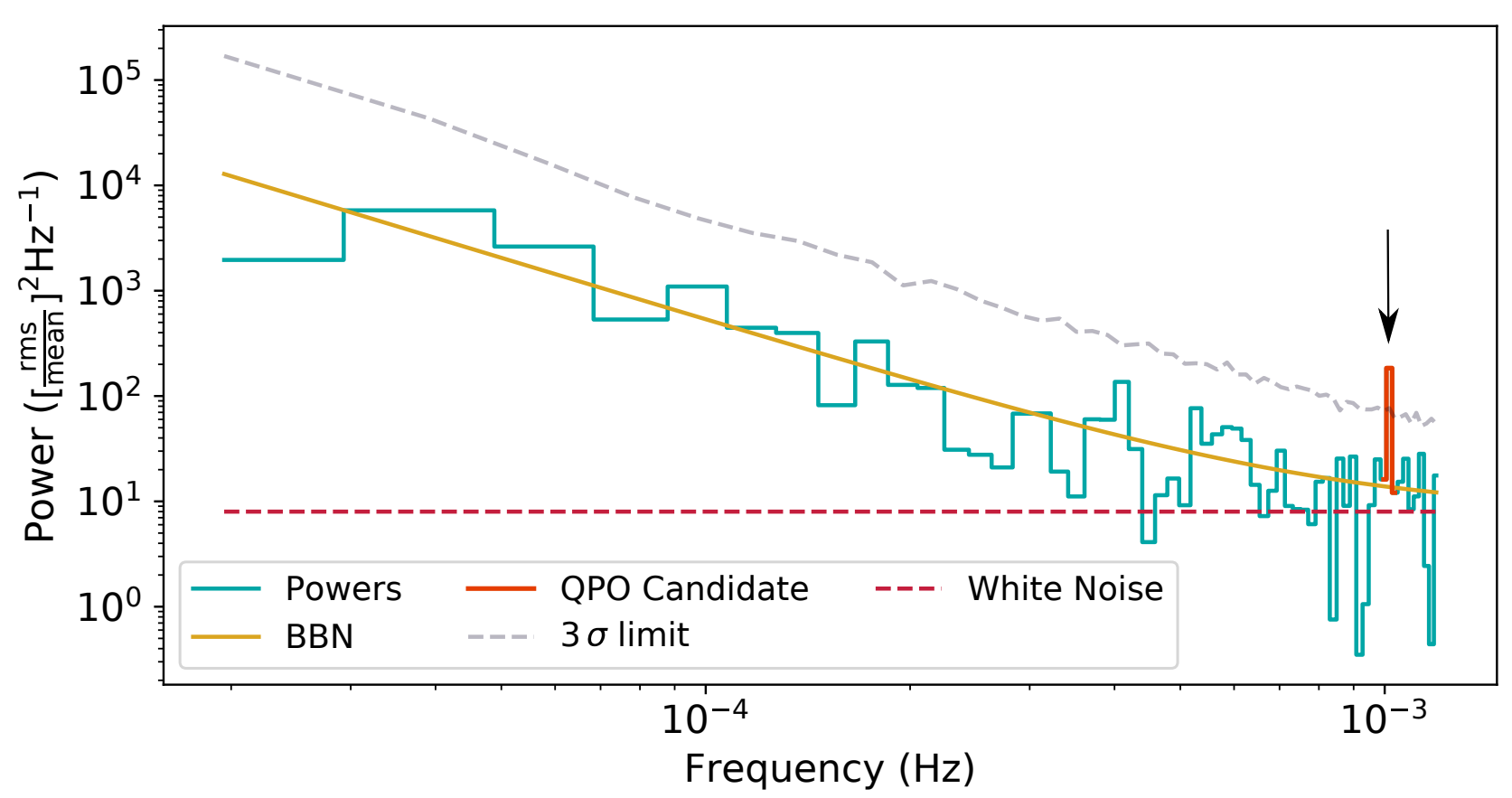

Figure 9. The highest significance QPO candidate (in the 0.9 - 1.6 keV band) found using the PLC model, in OBSID:0792180501 of IRAS 13224-3809. This standout candidate is observed with Q $>50$ and is one of only two examples we have found of a QPO candidate at $>1 \times 10^{-3} \mathrm{~Hz}$. See Table 1 for supplementary information.

energy band $(4.0-5.4 \mathrm{keV})$, but in others the index above the break is forced to be so steep as to bring the white noise cutoff down such that it would class as 'white noise dominated' by our definition.

- OBSID:0653510601. Similar to OBSID:0653510301, we find the detection in the peak significance energy band $(5.6-8.2 \mathrm{keV})$, remains above the $3 \sigma$ threshold, but the periodogram is at the limit of being white noise dominated.

Frequency-restricted search: Based on our QPO candidate detections, we proceed to search the other observations of 1H 0707-495 without blind detections at the modal QPO candidate frequency of $1.95 \times 10^{-4} \mathrm{~Hz}$. We detect a broad peak of power spanning this frequency in OBSID:0506200501 in the energy range of 4 $9 \mathrm{keV}$, with a frequency-restricted p-value $>3 \sigma$ and an outlier with a frequency-restricted $>3 \sigma$ p-value in OBSID:0653510601 at this same frequency. The latter is of particular interest, as in OBSID:0653510601 we observe a borderline $3 \sigma$ global significance detection at $1.27 \times 10^{-4} \mathrm{~Hz}$, which is strongest in the 5.6-8.2 keV energy range, but here we find the strongest frequency-restricted candidate at $3.4-4.0 \mathrm{keV}$, which extends to higher energies with reduced significance. It appears this feature may be similar to those QPO candidate detections in OBSIDs:0653510301 and 0653510501, but is intrinsically weaker and becomes dominated by a candidate at $1.27 \times 10^{-4} \mathrm{~Hz}$ at higher energies.

Previous claims: We note previous claims of significant QPO detections in 1H 0707-495, both concerning a single observation, OBSID:0511580401. Both Pan et al. (2016) and Zhang et al. (2018) report a QPO at $2.6 \times 10^{-4} \mathrm{~Hz}$ in the full $0.2-10 \mathrm{keV} \mathrm{XMM-Newton}$ energy range, via a Fourier and a non-Fourier wavelet analysis re- spectively. We do not recover a $3 \sigma$ result at this frequency, instead finding a $2 \sigma$ outlier at energies $>2.2 \mathrm{keV}$. We note that our blind selection criteria leads us to explore an 88.9 ks flare-subtracted segment in this observation, whereas Pan et al. (2016) opt instead to focus on the first $\sim 55 \mathrm{ks}$. This selection maximises their QPO significance, whilst considering the full observation reduces it to $90 \%$, well below the $3 \sigma$ detection threshold. We will revisit the impact of such segment selection on QPO detection in a forthcoming paper.

\subsection{PG 1244+026}

\subsubsection{Initial PLC search}

From a total of $7 X M M$-Newton observations of the NLS1 PG $1244+026$, we detect only a single $\geq 3 \sigma$ QPO candidate, within the $71 \mathrm{ks}$ longest continuous segment of OBSID:0675320101. We detect a QPO candidate at a frequency of $2.15 \times 10^{-4} \mathrm{~Hz}$ (see Figure 13), similar to the frequencies of those found in RE J1034+396,

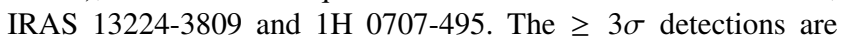
largely restricted to energies above $\sim 3 \mathrm{keV}$, as shown in Figure 6 . This differentiates it from RE J1034+396, IRAS 13224-3809 and $1 \mathrm{H} 0707-495$, which also show $\geq 3 \sigma$ detections at softer energies.

\subsubsection{Further tests}

\section{Test(i):}

- OBSID:0675320101. In all cases where the QPO candidate is determined to be $\geq 3 \sigma$, a weak break is found at $\sim 4 \times 10^{-5} \mathrm{~Hz}$. The QPO candidate remains highly significant in the $\sim 2.0-7.0 \mathrm{keV}$ energy range, with reduced significance towards higher energies, 


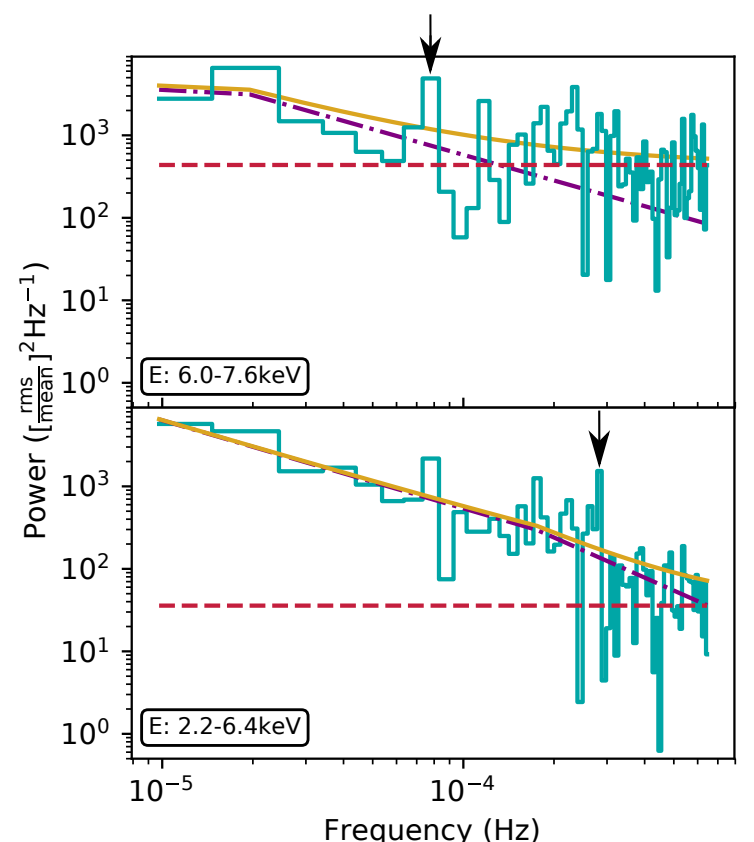

Figure 10. Energy resolved periodograms of IRAS 13224-3809 OBSID:0780561301, fitted with BKNPLC models following test (i). Top: periodogram in the $6.0-7.0 \mathrm{keV}$ band, with the QPO candidate highlighted at $7.81 \times 10^{-5} \mathrm{~Hz}$. We find fitting with this model causes the QPO candidate to drop to a $2 \sigma$ significance. Bottom: periodogram in the $2.2-6.4 \mathrm{keV}$ band, with the QPO candidate highlighted at $2.83 \times 10^{-4} \mathrm{~Hz}$, which remains $>3 \sigma$ before a steep drop in power at higher frequencies. Note: this frequency lies within the white noise in the higher energy band periodogram (top panel).

e.g. $2.0-9.0 \mathrm{keV}$. However, in multiple energy bands, the QPO candidate now falls above the white noise cutoff or the number of frequencies becomes too small to avoid being white noise dominated by our definition.

\section{Test(ii):}

- OBSID:0675320101. We find the power spectrum may be well described with a lower index of -1.1 and the results from this test match those of test (i).

Frequency-restricted search: We proceed to set an a-priori QPO candidate frequency (this time at $2.15 \times 10^{-4} \mathrm{~Hz}$ ), and repeated the analysis for all observations of PG $1244+026$. We find a single frequency-restricted $\geq 3 \sigma$ candidate at this frequency in OBSID:0744440301, strongest in the $2.0-7.8 \mathrm{keV}$ band.

\subsection{NGC 4051}

As with the cases of IRAS 13224-3809 and 1H 0707-495, NGC 4051 is one of best-studied, highly variable NLS1 known (e.g. Breedt et al. 2010, Vaughan et al. 2011).

\subsubsection{Initial PLC search}

Across 17 XMM-Newton observations, we detect QPO candidates at $\geq 3 \sigma$ significance in 3 . However, we report only two candidates

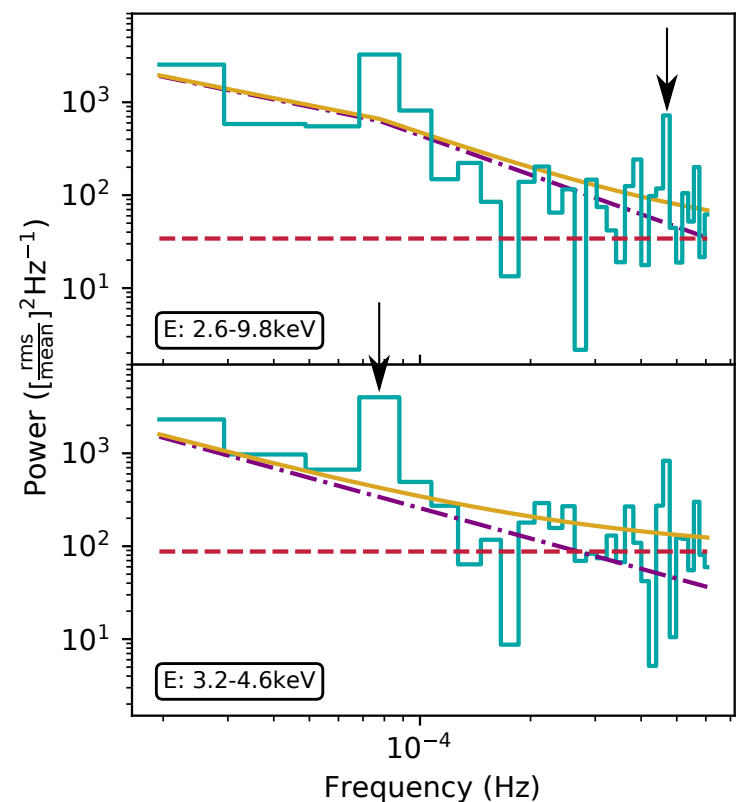

Figure 11. Energy resolved periodograms of IRAS 13224-3809 OBSID:0780561401, fitted with BKNPLC models following test (i). Top: periodogram in the $2.6-9.8 \mathrm{keV}$ band, with the QPO candidate highlighted at $4.69 \times 10^{-4} \mathrm{~Hz}$, found at a $>3 \sigma$ significance using a BKNPLC model, with the break located at $7.81 \times 10^{-5} \mathrm{~Hz}$. Bottom: periodogram in the 3.2 $4.6 \mathrm{keV}$ band; the QPO candidate is now found at the position of the break (contrast with the top panel) at $7.81 \times 10^{-5} \mathrm{~Hz}$. In this energy band, a break does not well describe the data and, following test (i), we find the QPO candidate at $7.81 \times 10^{-5} \mathrm{~Hz}$ reaches a $>3 \sigma$ significance. Note: the previous QPO candidate at $4.69 \times 10^{-4} \mathrm{~Hz}$ is beyond the white noise cutoff in this energy band (contrast with the top panel).

(see Table 1), at frequencies of $3.52 \times 10^{-4} \mathrm{~Hz}$ and $1.56 \times 10^{-4}$ $\mathrm{Hz}$ respectively. The former is shown as an example in Figure 1, and both candidates are compared directly in Figure 14. Notably, the QPO candidates in these observations are significant to $\geq 3 \sigma$ in energy ranges of $4.6-7.6 \mathrm{keV}$ and $3.2-5.4 \mathrm{keV}$ respectively (as shown in Figure 6). Hence, we appear to observe both frequency and slight energy variation between these candidates. We regard the detection of $\mathrm{a} \geq 3 \sigma$ candidate in observation OBSID:0606320301, (with a peak significance of 0.9979 , in the energy range $3.6-6.8 \mathrm{keV}$, and at a frequency of $1.56 \times 10^{-3} \mathrm{~Hz}$ ) to not be reliable as it sits in the highest Fourier frequency bin on the white noise cutoff.

\subsubsection{Further tests}

Test(i):

- OBSID:0109141401. A weak break at $\sim 1 \times 10^{-4} \mathrm{~Hz}$ is identified in most energy ranges, and in each case the QPO candidate remains $\geq 3 \sigma$. In a few energy ranges - namely narrower bands such as $5.0-6.0 \mathrm{keV}$ and $5.2-6.2 \mathrm{keV}$ - the observations now class as white noise dominated.

- OBSID:0606321801. A break is found consistently at $\sim 1 \times$ $10^{-4} \mathrm{~Hz}$, across all energy bands, close to the QPO candidate at $1.56 \times 10^{-4} \mathrm{~Hz}$. The large offset of power above the break has led to identification of a QPO candidate when using the PLC model, but as this is well described by a break, the candidate significance is now 


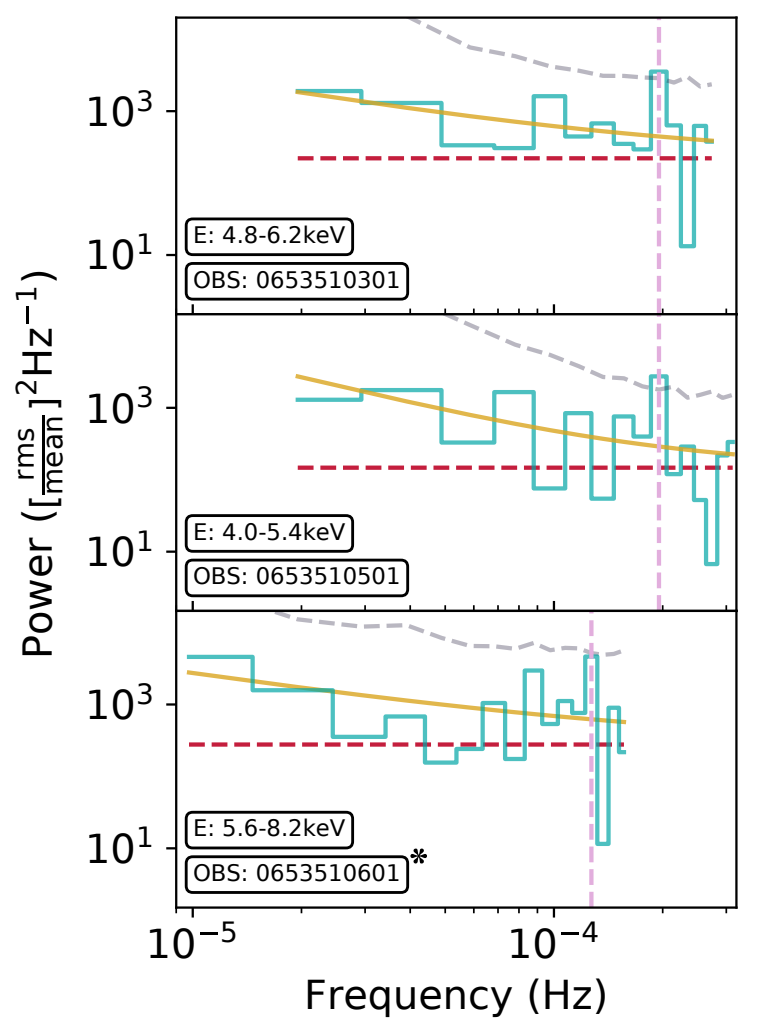

Figure 12. The highest significance $(\geq 3 \sigma)$ QPO candidates detected in $1 \mathrm{H}$ 0707-495 using the PLC model, with the corresponding frequency bin highlighted. As with RE J1034+396 and IRAS 13224-3809, we note the consistency of the QPO candidate frequency across multiple observations. We highlight borderline $3 \sigma$ results $(*)$.

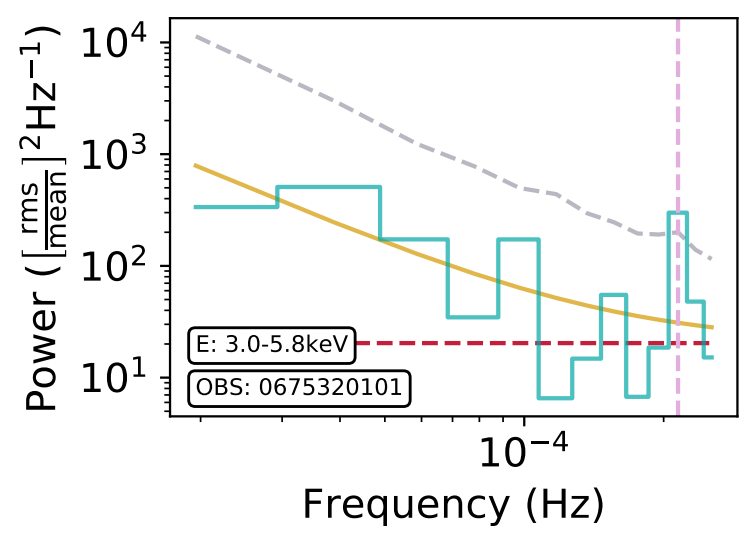

Figure 13. The highest significance $(\geq 3 \sigma)$ QPO candidate detected in a single observation of PG 1244+026 using the PLC model, with the corresponding frequency highlighted. lowered to a borderline $3 \sigma$ detection. Clearly this feature is data limited and requires further investigation.

\section{Test(ii):}

- OBSID:0109141401. The power spectrum of this observation is well described with a lower index of -1.1. This leads to results almost identical to those from test (i), returning a QPO candidate well above the $3 \sigma$ threshold for those energy bins not deemed to be white noise dominated.

- OBSID:0606321801. Unlike OBSID:0109141401, the power spectrum is not well described with a lower index of -1.1 , and the magnitude of the break is reduced near the QPO candidate frequency. In this case, the excess of power around the break is sufficient for the QPO candidate to remain detected at $>3 \sigma-$ highlighting the importance of model selection.

Frequency-restricted search: With two QPO candidate frequencies, it is difficult to motivate a single $a$-priori frequency for a search across those observations without blind detections. Searching at the two frequencies reported in Table 1, we find no supporting evidence of a feature at $1.56 \times 10^{-4} \mathrm{~Hz}$, but we do find a frequency-restricted $\geq 3 \sigma$ candidate at $3.52 \times 10^{-4} \mathrm{~Hz}$ in OBSID:0606320301, strongest at $3.6-5.0 \mathrm{keV}$, similar to the case of OBSID:0109141401.

Previous claims: NGC 4051 has been the subject of multiple X-ray variability studies over several decades, with Papadakis \& Lawrence (1995) reporting a broad QPO-like feature in EXOSAT data at a frequency of $\sim 4 \times 10^{-4} \mathrm{~Hz}$, which appears stronger in their low-energy band $(0.05-2) \mathrm{keV}$, than their medium-energy band $(2-10) \mathrm{keV}$. It should be noted that their methodology does not include the simulation of red noise following Timmer \& Koenig (1995), but does consider fitting with complex models. They find a PLC + GaussIAN model provides a good description of the underlying power spectrum and QPO-like feature at low energies, with a highly significant improvement over a PLC model. We note that the QPO candidate we observe in OBSID:0109141401 lies at a similar frequency $\left(3.52 \times 10^{-4} \mathrm{~Hz}\right)$, but is significant at harder energies within their medium-energy band (see Figure 6).

Green et al. (1999) study two, 0.1 - $2 \mathrm{keV}$ ROSAT observations of NGC 4051 and suggest the possible presence of a QPO-like feature at frequencies $>\sim 10^{-3} \mathrm{~Hz}$, but this is above the white noise limit for our observations. Lastly, Vaughan et al. (2011) conducted a variability analysis of NGC 4051 using a series of XMM-Newton observations (with a total of $\sim 570 \mathrm{ks}$ ), across the full energy range of $0.2-10 \mathrm{keV}$, and across a frequency range of $\sim 1 \times 10^{-4}-\sim$ $1 \times 10^{-2} \mathrm{~Hz}$. They report no QPOs, although they do note that their analysis at harder energies $(2-10 \mathrm{keV})$ provided tentative evidence for a possible feature at $\sim 4 \times 10^{-3} \mathrm{~Hz}$.

\subsection{ARK 564}

ARK 564 is another well-studied NLS1, both on short (e.g. Kara et al. 2017) and long (e.g. McHardy et al. 2006) timescales, providing deeper insights into the complex nature of the broad-band noise, which we discuss in Section 5.

\subsubsection{Initial PLC search}

We detect QPO candidates in 4 out of 11 XMM-Newton observations. The nature of these candidates appears less stable than in the 


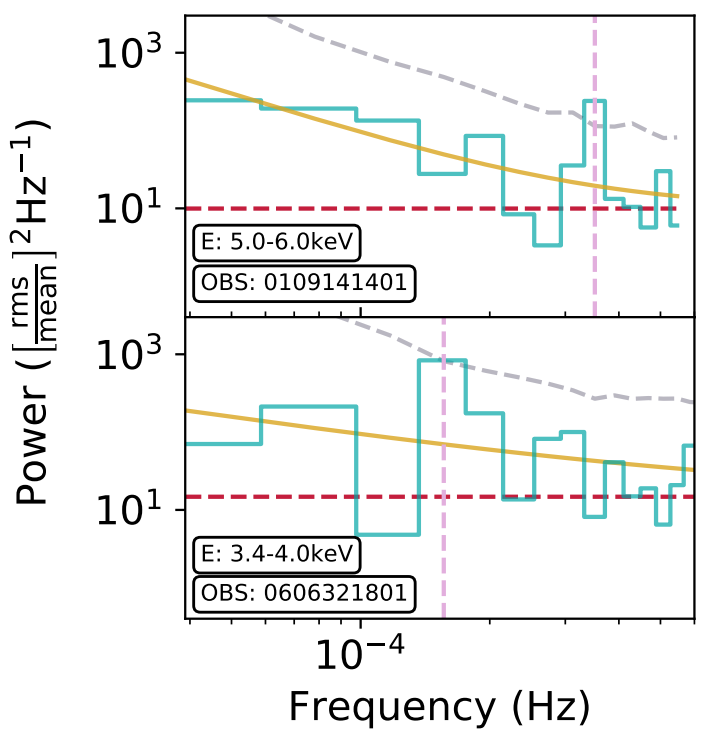

Figure 14. The highest significance $(\geq 3 \sigma)$ QPO candidates detected in NGC 4051 using the PLC model, with the corresponding frequency bin highlighted. We highlight those which are not validated by our further tests $(\dagger-$ see Section 3.2).

other AGN in our sample, and Figure 15 demonstrates the apparent movement of the QPO candidate towards lower frequencies with time.

The first observation in which we find $\mathrm{a} \geq 3 \sigma$ outlier (OBSID:0006810101) is similar to the higher frequency QPO candidate we have located in IRAS 13224-3809, at a frequency of $1.88 \times 10^{-3}$ $\mathrm{Hz}$. The longest segment in this observation is uncharacteristically short, at only $4.95 \mathrm{ks}$, but with a period of $\sim 532 \mathrm{~s}$ we can still obtain a moderate quality factor of $Q \approx 6$. In OBSID:0670130501, we detect a QPO candidate at a frequency of $8.59 \times 10^{-4} \mathrm{~Hz}$, prior to the observations of OBSID:0670130701 and OBSID:0670130801 in which we find QPO candidates at $2.73 \times 10^{-4} \mathrm{~Hz}$ and $2.54 \times 10^{-4}$ $\mathrm{Hz}$ respectively. The energy ranges in which these four QPO candidates are detected are largely consistent, being dominated by counts at $2-3 \mathrm{keV}$. The full range of candidate significance as a function of energy is shown in Figure 6.

\subsubsection{Further tests}

Test(i):

- OBSID:0006810101. In requiring a break to occur at or before the QPO candidate, we find every energy bin in this observation to be considerably restricted in the number of available frequency bins below the white noise cutoff. As a result, we cannot discern the significance of this QPO candidate. As such, this detection is dependent on our selection of white noise cutoff, so as a result it difficult to discern from white noise and is brought into question.

- OBSID:0670130501. We find a clear break at a frequency of $3 \times 10^{-4} \mathrm{~Hz}$ above $2 \mathrm{kev}$, and find a break at $5 \times 10^{-4} \mathrm{~Hz}$ when including energies below $2 \mathrm{keV}$. In the case of the former, we find the QPO candidate at $8.59 \times 10^{-4} \mathrm{~Hz}$ to increase in significance. In those energy bands where the break is located at $5 \times 10^{-4} \mathrm{~Hz}$, the significance of the QPO candidate is reduced, but remains around $3 \sigma$.

- OBSID:0670130701. We find a break at a frequency of $8 \times$ $10^{-5} \mathrm{~Hz}$ in the $2.4-3 \mathrm{keV}$ energy range, with the break moving to $1 \times 10^{-4} \mathrm{~Hz}$ for $2.6-3.0 \mathrm{keV}$ and $2 \times 10^{-4} \mathrm{~Hz}$ for $2.6-3.2 \mathrm{keV}$ - with the QPO candidate at $2.73 \times 10^{-4} \mathrm{~Hz}$. The QPO candidate remains $>3 \sigma$, but decreases in significance with increasing energy and a higher break frequency. We also find the peak significance band (2.6 - $2.8 \mathrm{keV})$ now classes as white noise dominated as the BKNPLC invokes a cutoff at a lower frequency.

- OBSID:0670130801. This observation contains the strongest QPO candidate we have located in ARK 564. Once again, the break is highly energy dependent: a break is found at $\sim 1 \times 10^{-4} \mathrm{~Hz}$ in the $3.0-4.0 \mathrm{keV}$ energy range, but a strong break is instead found at $\sim 6 \times 10^{-5} \mathrm{~Hz}$ for $5.0-10.0 \mathrm{keV}$, before the power spectrum flattens for energy bands $>6 \mathrm{keV}$, resulting in a weak break. In each case, the QPO candidate is found above the $3 \sigma$ threshold, although the significance lessens in energy bands $>6 \mathrm{keV}$.

\section{Test(ii):}

- OBSID:0006810101. All energy bands are classified as white noise dominated assuming this model.

- OBSID:0670130501. A lower index of -1.1 does not provide a good description for the break across the full range of energy bands. This model typically locates the break at $6 \times 10^{-4} \mathrm{~Hz}$ and reduces the QPO candidate significance, although not below the $3 \sigma$ threshold.

- OBSID:0670130701. Again, a lower index of -1.1 does not allow for a good description of the periodogram, but the QPO candidate remains above the $3 \sigma$ threshold.

- OBSID:0670130801. As with the other observations, the periodograms are not well described with a lower index of -1.1. As a break is not preferred, most QPO candidates remain above the $3 \sigma$ threshold, with the exception of energy bands $>5.6 \mathrm{keV}$, in which the candidates lie at the white noise cutoff frequency.

Frequency-restricted search: The observed variation in QPO candidate frequency means we have minimal a-priori knowledge of a stable frequency for deeper searches, and therefore we exclude this step for ARK 564.

Previous claims: McHardy et al. (2007) reported an excess of power in the $2.0-8.8 \mathrm{keV}$ band in OBSID:0206400101 at $\sim 2 \times 10^{-4} \mathrm{~Hz}$ - similar to two of our QPO candidates in OBSID:0670130701 and OBSID:0670130801 - but only at a 90\% significance. Our analysis of this observation does not reproduce the feature, as the longest continuous segment of this $\sim 101 \mathrm{ks}$ observation is only $\sim 21 \mathrm{ks}$, severely limiting our frequency range.

\subsection{MRK 766}

MRK 766 is again well-studied (e.g. see Markowitz et al. 2007), with nine XMM-Newton observations in the archive at the time of writing.

\subsubsection{Initial PLC search}

We report a single QPO candidate at $\geq 3 \sigma$, in a single energy bin, $3.0-4.6 \mathrm{keV}$, as shown in Figure 16. Figure 6 demonstrates how the QPO candidate significance drops below the $3 \sigma$ threshold in 


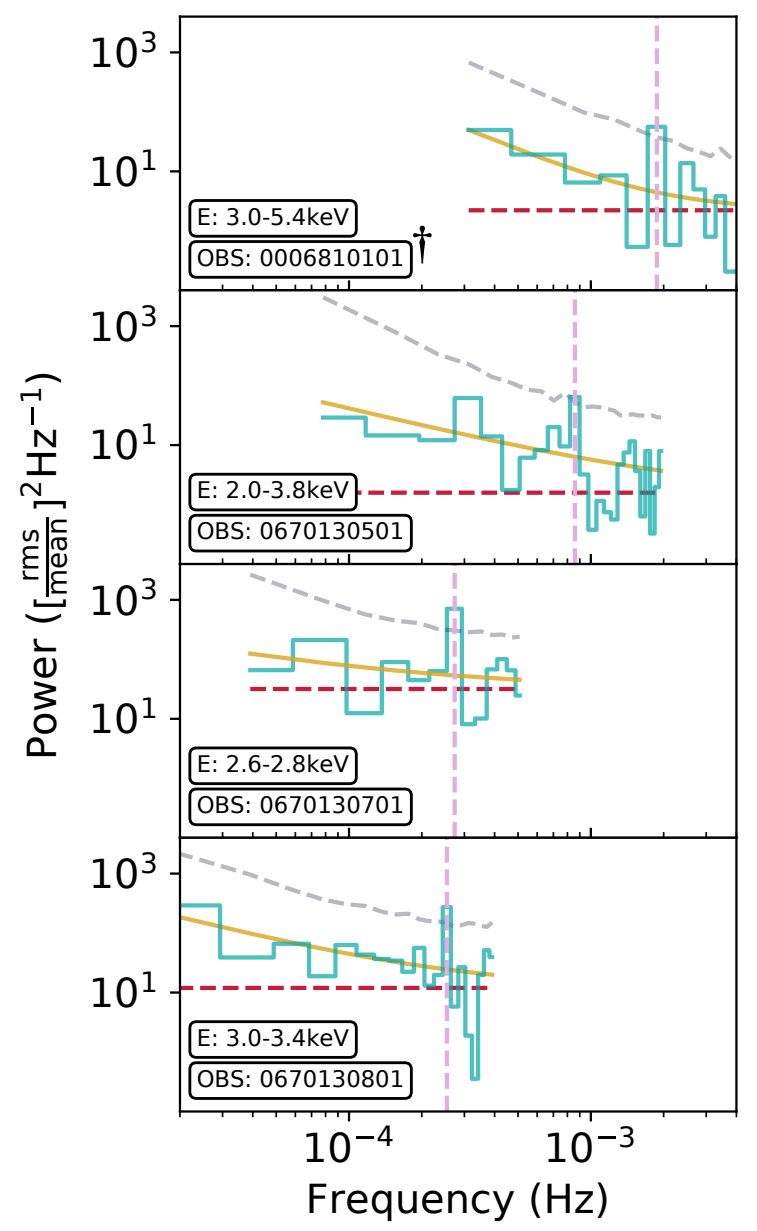

Figure 15. The highest significance $(\geq 3 \sigma)$ QPO candidates detected in ARK 564 using the PLC model, with the corresponding frequency bin highlighted. We note the apparent shift of the candidate to lower frequencies across each observation (and with time), where it appears to stabilise. We highlight those which are not validated by our further tests $(\dagger-$ see Section 3.2).

surrounding energy bins. At a frequency of $4.69 \times 10^{-4} \mathrm{~Hz}$, the QPO candidate frequency is similar to that of the other candidates we have located in our sample.

As our significance tests are based on an underlying stochastic background, it is quite possible we may determine an outlier to lie very close to $3 \sigma$ (peaking above or dipping below this threshold under repeated tests). For our analysis of MRK 766, this occurs for our only candidate. This borderline $3 \sigma$ detection, coupled with a lack of supporting evidence from other observations (e.g. in IRAS 13224-3809 and 1H 0707-495 we have found multiple QPO candidates across multiple observations at the same frequency across similar energy ranges) means we remain sceptical of this detection, however we report the findings to allow a comparison to be made to future studies.

\subsubsection{Further tests}

\section{Test(i):}

- OBSID:0304030301. We find a tentative break at $\sim 1 \times 10^{-4}$

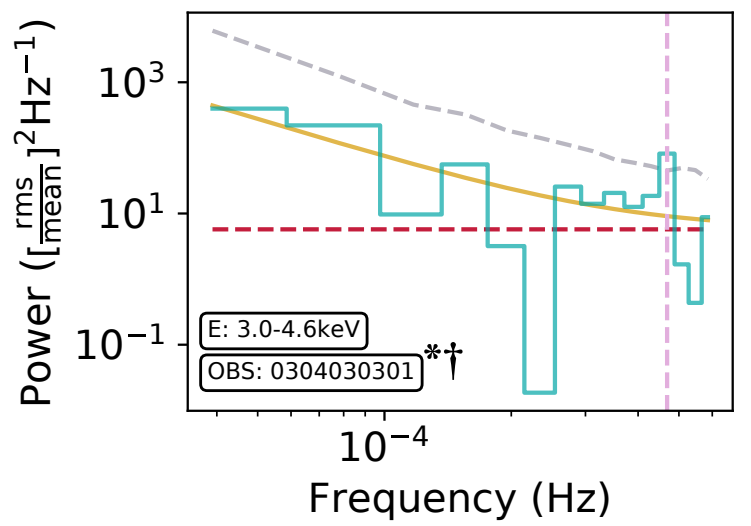

Figure 16. The highest significance-borderline-QPO candidate detected in the single observation of MRK 766 using the PLC model, with corresponding frequency bin highlighted.

$\mathrm{Hz}$ and white noise cutoff at $\sim 5 \times 10^{-4} \mathrm{~Hz}$, which heavily restricts the number of frequency bins such that the periodograms are defined as white noise dominated.

\section{Test(ii):}

- OBSID:0304030301. With a frozen lower index of -1.1 we obtain identical results to test (i).

\section{Frequency-restricted search:}

For completeness, we assume an a-priori $\mathrm{QPO}$ candidate frequency of $4.69 \times 10^{-4} \mathrm{~Hz}$, and searched within the other observations, finding a restricted-frequency $>3 \sigma$ candidate in OBSID:0096020101, strongest in the $0.7-1.2 \mathrm{keV}$ band - notably at a lower energy range than in OBSID:0304030301.

Previous claims: We note the previous claims of a strongly significant QPO in a single observation of MRK 766 (OBSID:0304030601) identified through a non-Fourier wavelet method (Zhang et al. 2017). However, we do not find a significant feature at their reported frequency of $1.55 \times 10^{-4} \mathrm{~Hz}$.

\subsection{Observations as free trials}

The significance values we have quoted for each QPO candidate rely on the assumption that each observation is independent and the number of free trials is limited to only the frequency range of interest, per observation. Our motivation for not treating each observation as being a free trial stems from the observation that certain spectral states, connected to the accretion rate, seem to be necessary for such QPOs to appear (e.g. Alston et al. 2014).

We can instead employ the most conservative perspective and assume each observation (200 in total) to be a free trial - regardless of data quality - and obtain the most stringent QPO candidate significance.

In the case of QPO candidates present at a single frequency in a single observation, such as PG $1244+026$ and NGC 4051, we find the significance values fall to $<3 \sigma$. In the case of NGC 4051 OBSID:0109141401, this is reduced to the $\sim 2 \sigma$ level, while PG $1244+026$ OBSID:0675320101 falls to $<2 \sigma$. However we note that 
such decreases are overestimates of the impact (as we have assumed the data quality to be equivalent in all observations).

For those AGN with QPO candidate detections at the same frequency across multiple observations, we consider both the more stringent 'look elsewhere' effect set against the conditional probability of detecting a QPO candidate at the same frequency. Combining the two leads to the QPO candidate in $1 \mathrm{H} \mathrm{0707-495}$ being at $>6 \sigma$ significance, while the IRAS 13223-3809 QPO candidate at a frequency of $9.77 \times 10^{-5} \mathrm{~Hz}$ may similarly be found at $>6 \sigma-$ although this drops to $>4 \sigma$ if excluding the detection in OBSID:0792180301, where the broad-band noise is not well constrained. In the cases of ARK 564 and RE J1034+396, our global significance values for some candidates of interest reach $G S=1.0000$, whereby we reach a precision limit for 10,000 MC simulations. If instead we consider these individual global significance values to be similar to those observed in $1 \mathrm{H} 0707-495$, we find the $2.54-2.73 \times 10^{-4} \mathrm{~Hz}$ candidate in ARK 564 to be $>4 \sigma$, whilst RE J1034+396 reaches the $>7 \sigma$ level, with four observations all at equivalent frequencies. We note that these values in themselves are conservative, as they consider only $>3 \sigma$ global significance values in Table 1 , and no supplementary evidence from observations with lower significance candidates.

\section{ADDITIONAL UNCERTAINTY IN THE BROAD-BAND NOISE}

We have chosen to focus on relatively simple models for the broad band noise as these are typically a good description in the frequency range we are studying (e.g. González-Martín \& Vaughan 2012). However, there is the potential for further underlying complexity in the broad band noise and leakage which can effect our QPO candidate significance claims and which we explore here.

\subsection{The impact of the white noise cutoff}

Determining the frequency at which the underlying white noise (Poisson noise) within the observation becomes dominant over the intrinsic red noise is subject to inherent uncertainty in any fitting of the broad-band noise. In our approach to defining the null hypothesis (Section 3.1.1) - the QPO candidate is removed from the periodogram and the broad-band noise is re-fitted (see Vaughan 2005), but the white noise cutoff frequency is left unchanged from our fit where the QPO candidate is included. Defining this frequency is a somewhat complex issue as the periodogram is by definition only one realisation of the underlying power spectrum (and so would be best represented by a probability distribution) and the position of the cutoff naturally has an uncertainty, correlated with those uncertainties on the normalisation and power-law index (such that a different draw from the covariant distribution - Figure 2 - produces a different cutoff). As we are required to select a single cutoff frequency (otherwise we introduce an uneven bias into our significance testing), we opted to retain the value from our initial fit but now explore the impact of this choice.

Re-fitting the broad-band noise with the QPO candidate removed tends to reduce the white noise cutoff frequency. The result is that bins at high frequency may then be outside of our range of interest. As we also require 13 Fourier bins (which includes the QPO candidate bin - although this frequency is ignored when the null hypothesis is defined) for fitting purposes, this can have the knock-on effect of removing energy bands from our study. The

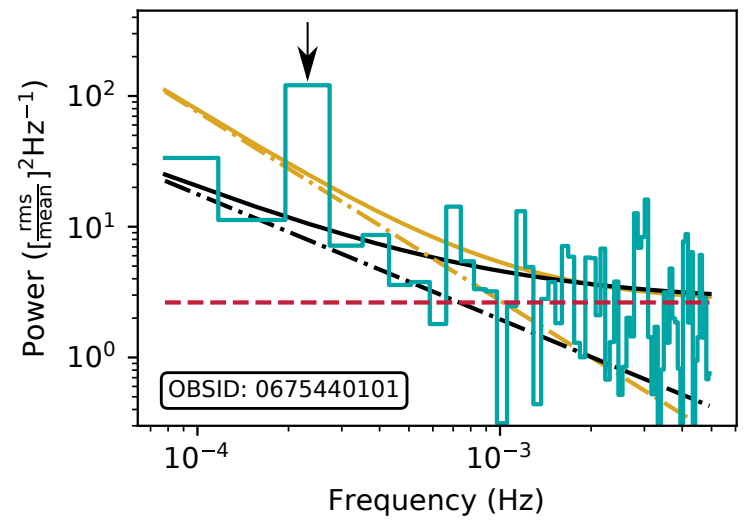

Figure 17. Example of the impact of the white noise cutoff selection via our two approaches (see Section 5.1), for the established QPO in RE J1034+396, OBSID: 0675440101 (within the most significant energy band, see Table 1). We show two PLC model fits, one (gold) including the QPO candidate bin, and the other (black) without the QPO candidate bin. The respective white noise cutoff frequencies in each case are determined to be the point where the underlying PLC model (dot-dashed) intersects the constant white noise level. As shown here, excluding the QPO candidate in our fitting results in the frequency of the white noise cutoff being lowered on average by $\sim 2-3$ bins. As we demonstrate in this example, we then risk reducing the number of bins in our periodogram to below the minimum requirement we have set for modelling the broad band noise - this would lead us to exclude this established and accepted QPO.

combination of these two effects can remove QPO candidates either because they now fall into the white noise dominated region or because there are too few bins for reliable fitting. We investigated these effects by setting the white noise cutoff frequency from the PLC model applied to the data without the QPO candidate. We find that $\sim 39 \%$ of the $>3 \sigma$ energy bands are now white noise dominated (with $<13$ frequencies in the periodogram, or 12 bins when excluding the QPO candidate bin), a change driven by the removal of those narrow energy bands with relatively low count rates and so large amounts of white noise, which were close to this boundary in our original analysis. Despite the loss of numerous energy bands, the majority of our reported $>3 \sigma$ QPO candidates remain, with a few exceptions, namely: IRAS 13224-3809 OBSIDs: 0780561301 $\left(v=7.81 \times 10^{-5} \mathrm{~Hz}\right.$ only), 0792180301,$0792180601 ; 1 \mathrm{H} 0707-495$ OBSID:0653510301, ARK 654 OBSID:0006810101, MRK 766 OBSID:0304030301, and perhaps most notably: RE J1034+396 OBSID:0675440101 (as well as almost all energy bins of OBSID:0655310201). It should be noted that a number of these observations were already borderline/close to the white noise threshold.

We note that, although setting the white noise cutoff to the higher of the two frequencies is a more conservative approach, it also has the potential to remove real signals, as is evident for the case of RE J1034+396 (see Figure 17). In a similar fashion, the QPO candidate of 1H 0707-495 in OBSID:0653510301, is discarded by this more stringent analysis but is still observed in OBSID:0653510501 at the same frequency, and so we find we can lose high probability detections. 


\subsection{The effect of windowing/red noise leak}

As we are only selecting a relatively small frequency range compared to the full extent of the underlying power spectrum (see e.g. Uttley et al. 2002, McHardy et al. 2006), we may expect some leakage of power into our observed band (see González-Martín \& Vaughan 2012). In Fourier space this is equivalent to convolving the Fourier transform of the window function (the Fejér kernel) with the underlying power spectrum (van der Klis 1988). As a result, the intrinsic broad-band noise may not be well represented by that which we measure in our periodogram. We explore the impact of leakage on those periodograms in which we have significant QPO candidate detections, by simulating a lightcurve based on an underlying power-law with an index of -2 following Timmer \& Koenig (1995) but extending back to $10^{-5} \mathrm{~Hz}$, i.e. to lower frequencies than we typically observe down to, and then apply a window equivalent to our segment selection. To obtain an appropriate normalisation for our input power-law, we first create very long simulated lightcurves (far longer than the real data) from a power-law with an index of -2 and trial normalisation stepping over log space. From these lightcurves we select a random segment of the same length as our real data and minimise the log-likelihood when compared to our observed periodogram (by minimising the median of the distribution of $S$ values). Using this best-fitting normalisation - which is different for each QPO candidate-detected observation - we simulate 10,000 fake lightcurves, apply our window, obtain the periodogram, and search for false outliers. We note this to be a somewhat extreme test as it is quite likely that a break to to flatter indices occurs before such low frequencies are reached (see e.g. McHardy et al. 2007) or that the power-spectrum itself is flatter $(\beta>-2)$ as we move to higher energies (Jin et al. 2020; Ashton et al. in prep.) where our QPO candidates are mostly located. In Figure 18 we present an example of our test for the impact of windowing as applied to NGC 4051 (OBSID:0109141401), where we plot the intrinsic noise needed to create the observed noise.

We apply the above test to only the most significant energy-resolved QPO candidates (as listed in Table 1) and find that 8 out of the 24 candidates remain above the $3 \sigma$ threshold. These include RE J1034+396 (OBSIDs: 0506440101 and 0675440201), IRAS 13224-3809 (OBSIDs: 0780561401 and 0792180501), NGC 4051 (OBSID: 0109141401) and ARK564 (OBSIDs: 0006810101, 0670130701 and 0670130801). The remainder lie above a $\sim 98 \%$ threshold, i.e. an upper $2 \sigma$ level. This is a clear indication that under extreme conditions it is possible for red noise leak to have an impact on QPO detection. However, we note that this may not rule out the now $<3 \sigma$ candidates as, in many cases (e.g. RE J1034+396), we find the QPO candidate frequencies to remain similar across multiple observations, which is hard to reproduce through fake signals generated by an underlying stochastic process.

\subsection{Complex broad-band noise models}

A natural extension to a test using a BKNPLC model is one in which the underlying noise is described by a series of Lorentzians (e.g. McHardy et al. 2007). This makes sense physically where each radius acts as a low pass filter with variations suppressed on a local viscous timescale (e.g. Churazov et al. 2001; Ingram et al. 2016), although in reality these are well known to be coupled (Uttley et al. 2017). Whilst we do not have a-priori knowledge of the nature of the Lorentzians that might describe the shape of the broad band noise in any of our energy-dependent observations, we can test the impact
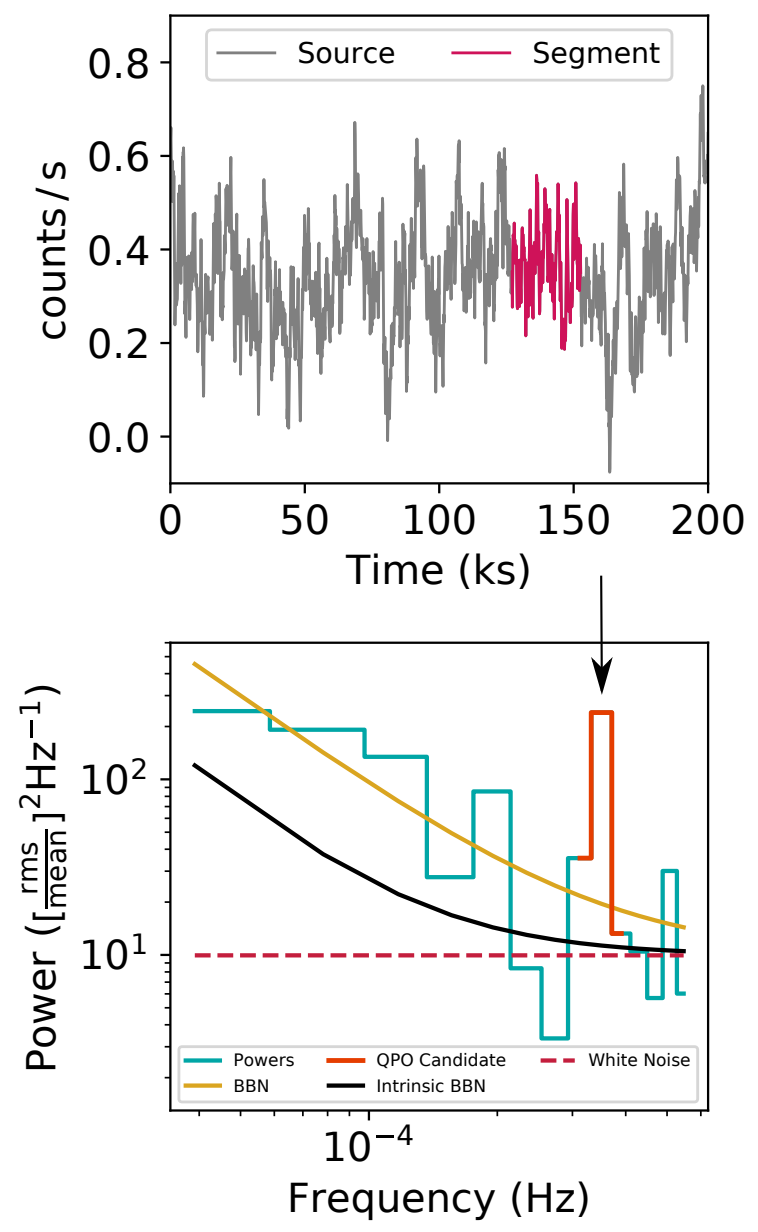

Figure 18. Top: example of a simulated light curve for NGC 4051 (OBS ID:0109141401), with a highlighted, randomly selected segment of length equal to the longest observed continuous segment of our real data. Bottom: observed periodogram (QPO highlighted), with best fitting PLC model and the intrinsic, pre-windowed PLC model.

such a model might have on QPO significance tests more generally. Assuming the high energy Lorentzians which describe the data of ARK 564 from McHardy et al. (2007) without white noise, we create fake periodograms down to $10^{-5} \mathrm{~Hz}$, a distribution of $R$ values, and obtain the free-trial-corrected $3 \sigma$ contours (see Vaughan 2005). We then proceed to fit the average of these simulated periodograms with our PLC model (minus white noise) and, using the best-fitting value for the index and normalisation, simulate again to obtain another set of $3 \sigma$ contours. As shown in Figure 19, by comparing the two sets of contours, we can see that, over much of the frequency range, the significance is under-estimated when a power-law model is used as an approximation, although the significance contours are comparable as the higher frequency Lorentzian starts to dominate the broad band noise.

Should a multiple Lorentzian model be a more appropriate description of the underlying noise for all of our observations at high energies, then the impact on our QPO candidate significance is expected to be relatively minor. However, given the lack of constraints on the nature and energy-dependence of any Lorentzians underlying our data, we stress that this is an open question and reiterate that 
accurately modelling the shape of the noise is vital for any robust significance claims (see Vaughan et al. 2016 for more discussion on this topic).

\section{DISCUSSION}

To-date, the presence of AGN X-ray QPOs across multiple observations has been limited to strong detections in the NLS1 AGN, RE J1034+396 (Gierlinski et al. 2008), with a single claim in MS 2254.9-3712 (Alston et al. 2015), a number of tentative claims in other NLS1s, and signals in TDEs (see Pasham et al. 2019b). Here we have performed the first energy-resolved search for QPO candidates within 38 AGN, comprising 200 XMM-Newton observations. We initially find 24 QPO candidates across 7 AGN, and subject to further tests, report the detection of 16 QPO candidates across 6 AGN (we exclude MRK 766 from this category - see Section 4.7).

Our results include the QPO in RE J1034+396 which we used to corroborate our approach, as well as confirming signals in IRAS 13224-3809, 1H 0707-495 and ARK 564 albeit it in some cases at different frequencies to those quoted in the past. In addition, we provide details of the energy dependence and the repeat occurrence of these signals across observations. This last point is important - in many cases (notably IRAS 133224-3809 and 1H 0707-495) the QPO candidates appear at the same or very similar frequencies (see Table 1); it is very difficult to create such repeat signals by chance alone, increasing the likelihood that they are genuine (see Section 4.8, where we reach significance levels in excess of $>6 \sigma$ for $3 \mathrm{AGN}$ ). We also note that, in most cases, the quality factor and rms of the signal is large (Q $>10$ and FRMS $\gtrsim 10 \%)$ in keeping with those established QPOs in RE J1034+396.

A possible limitation in our approach to estimating the significance of our QPO candidates, is our data-led maximum likelihood search for a break in the power spectrum, which does not incorporate any prior expectations we have for the position of the break (e.g. McHardy et al. 2007; González-Martín \& Vaughan 2012). At present, we merely force a break into the periodogram as a rigorous test of the QPO candidate significance (see Section 3.2); the occasional change in QPO candidate significance then underlines the need for caution when claiming the presence of a QPO, even when the broad-band noise model is statistically preferred. In future, and with data which extends to lower frequencies, we will be able to perform more stringent tests. We note that, whilst our approach may appear to discard information prior to our fitting, the present lack of a well-constrained probability distribution describing the position and energy dependence of the break prevents such an approach. Given the situation of poorly motivated priors and somewhat limited data, we have chosen to follow a frequentest approach, although Bayesian inference may allow for a more accurate assessment of the presence of breaks in future. Bearing in mind the above caveats and our warnings about the uncertainty in the broad-band noise, we proceed to discuss the QPO candidates our analysis has identified, and the possible unifying features in our sample.

RE J1034+396 is well known for having a strong soft excess (Puchnarewicz \& Soria 2002; Casebeer et al. 2006; Middleton et al. 2007, 2009), likely a result of extremely high accretion rates, which has led to comparisons being made between its QPO and the $67 \mathrm{~Hz}$ HFQPO observed in GRS 1915+105 when accreting at high Eddington fractions (Morgan et al. 1997; Ueda et al. 2009; Middleton \& Done 2010, Jin et al. 2020). More generally, HFQPOs are only seen in BHBs in their very high state (see Remillard \& McClintock 2006), when the mass accretion rates can reach significant fractions of their Eddington limit (e.g. see Nowak 1995). It is therefore reasonable to postulate that the HFQPO mechanism is intrinsically tied to these high mass accretion rates (Blaes et al. 2006).

Of our sample of AGN containing QPO candidates, RE J1034+396, IRAS 13224-3809, PG 1244+026, 1H 0707-495 and ARK 564 have all been reported accreting at close to their Eddington limits (Done \& Jin 2016; Alston et al. 2020b; Kara et al. 2017; Done et al. 2012b) as is also the case for MS 2254.9-3712 (Alston et al. 2015).

Indeed, NGC 4051 may be the only exception, accreting at 0.1 Eddington (Peterson et al. 2000; Alston et al. 2013). Whilst the uncertainty involved in mass accretion rate determination means we cannot be completely certain of the true nature of these sources, there could well be a link between the high accretion rates in these AGN (as well as in TDEs) and the QPO candidates we have found.

Naturally, high accretion rates (and therefore high luminosities) could result in some level of selection bias - our ability to detect a QPO relies on high signal-to-noise ratio data and so we may be more likely to detect signals in predominantly bright, high accretion rate AGN. We have been unable to test this in our present sample due to the uncertainty in SMBH mass measurements and therefore accretion rate estimates. It is quite possible however, that QPOs in AGN are far more commonplace than previously thought, but the present difficulty in obtaining the required data quality to probe a sufficient frequency range, biases our study towards intrinsically bright, or sufficiently local AGN. In future, new, high-throughput instruments such as STROBE-X and Athena will be able to probe many more AGN to higher frequency ranges.

The energy bands in which we have found significant QPO candidates vary between sources, but remain largely consistent between observations of the same source and, with the exception of RE J1034+396, the detections are most significant in bands above $2 \mathrm{keV}$. This was also found by Alston et al. (2015) in their investigation of MS 2254.9-3712, where the QPO is detected at 2-5 keV and provides further compelling evidence that the hard energy components (i.e. the coronal emission and reflection), of the AGN energy spectrum appear to be central to QPO production. The energydependence of detection significance also indicates why previous studies (e.g. González-Martín \& Vaughan 2012) have been unlikely to detect these QPO candidates, as they do not appear in the typically studied full bandpass $0.3-10 \mathrm{keV}$, nor generic soft, $0.3-2 \mathrm{keV}$, or hard, $2-10 \mathrm{keV}$ bands. In a forthcoming paper we will investigate the full energy-dependence and detailed nature of these candidates to search for confirmatory evidence of their QPO (i.e. non-broadband noise) nature, and thereby hope to better understand the QPO production mechanism in AGN.

The QPO frequency is naturally an important parameter in understanding the accretion flow (e.g. as a result of motion of the flow or characteristic timescale of an instability). Our prior knowledge of QPO frequencies in this regard was somewhat limited to the $\sim 2.5 \times 10^{-4} \mathrm{~Hz}$ detection in RE J1034+396 (Gierlinski et al. 2008 ) and the $\sim 1.5 \times 10^{-4} \mathrm{~Hz}$ detection in MS 2254.9-3712 (Alston et al. 2015). Here we have found QPO candidates with similar frequencies in 1H 0707-495 and PG 1244+026, whilst NGC 4051 and IRAS 13224-3809 have QPO candidates with comparable frequencies, but with greater variation between observations.

IRAS 13224-3809 is of particular interest for both the number of $\geq 3 \sigma$ candidates and the consistency of their frequencies, with many close to $\sim 1 \times 10^{-4} \mathrm{~Hz}$. The presence of possible harmonic features (the nature of which we will explore in more detail in the near future) is also unique amongst all of our observations, although this may simply be a consequence of being able to probe to higher 


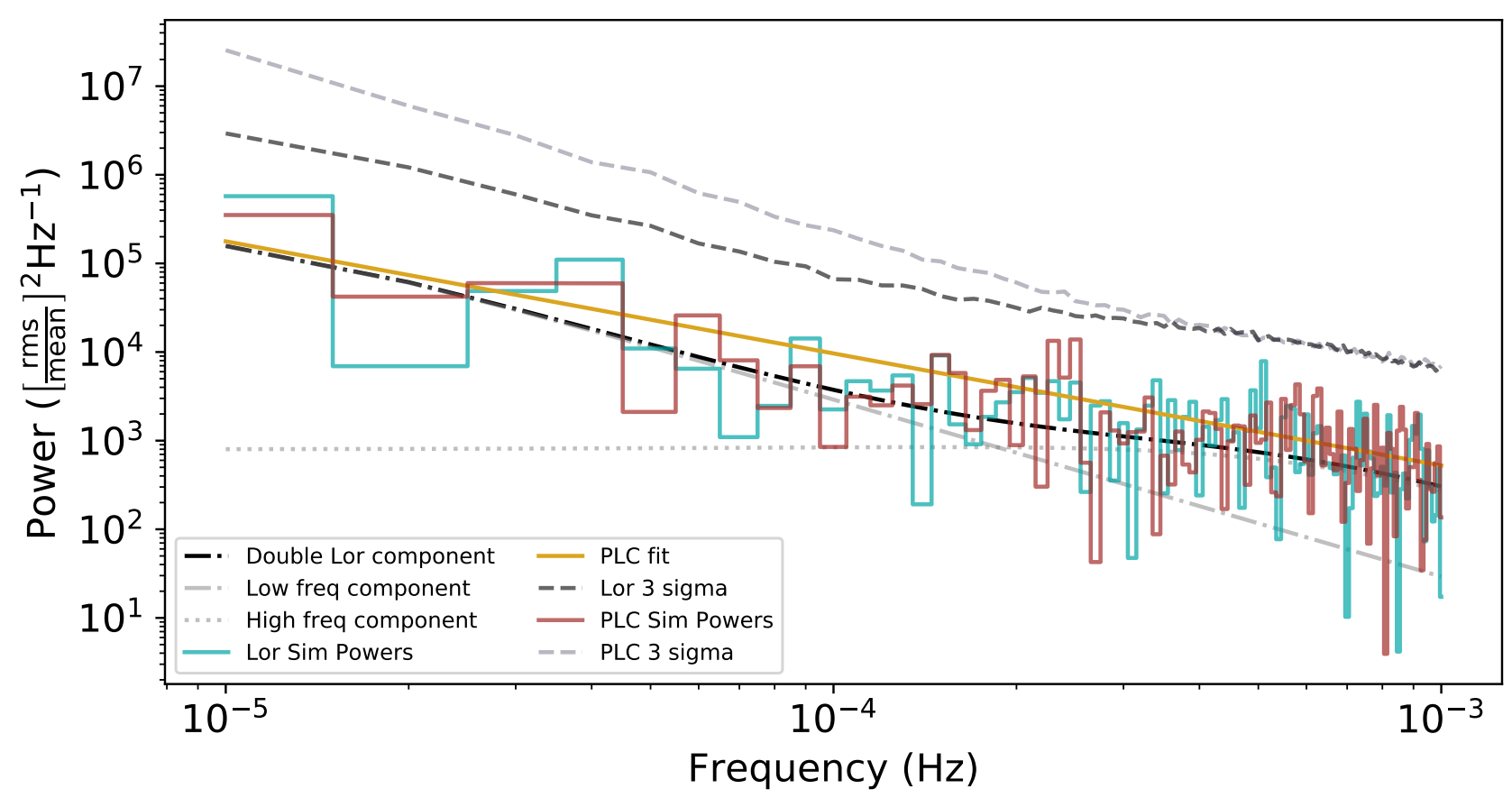

Figure 19. Simulated power spectra from $10^{-5}-10^{-3} \mathrm{~Hz}$, generated from the high energy, double Lorentzian model for ARK 564 (McHardy et al. 2007) and a PLC model fitted to the data generated from this. The model choice has a notable impact on the $3 \sigma$ significance contours (Vaughan 2005). In this case, should we assume a PLC model but a double Lorentzian model is the correct description of the broad-band noise, we would potentially underestimate QPO candidate significance at low frequencies.

frequencies due to the higher count rates in this AGN. As further indicated by the higher frequency QPO candidate detected in ARK $564\left(\sim 1 \times 10^{-3} \mathrm{~Hz}\right)$, being able to probe to high, non-white noisedominated frequencies (especially at higher energies) may reveal many more QPO candidates.

ARK 564 is rather unusual amongst our sample, with a QPO candidate frequency decreasing with time, before an apparent stabilisation around $\sim 2 \times 10^{-4} \mathrm{~Hz}$. This may be due to the changing frequency range we can probe, as the lowest frequency and white noise cutoff frequency also evolve across observations. Conversely, it may be the result of the physical process creating a genuine QPO changing with time, potentially settling, as suggested in the case of RE J1034+396 (Gierlinski et al. 2008). As noted earlier, these results assume a simplified description of the underlying broadband noise and, for ARK 564 specifically, previous modelling has identified the presence of breaks and Lorentzians (e.g.Papadakis et al. 2002, McHardy et al. 2007), which in one observation occurs close to our QPO candidate frequency (OBSID:0006810101). As we point out via simulations, it is unlikely that modelling the broad-band noise with Lorentzians could lead to an overestimate of QPO significance, however, as we have demonstrated, care should be taken when assuming the shape of the broad-band noise to be simple.

Although we will probe the detailed nature of these QPO candidates in a follow-up paper, we note that, should they be analogous to those HFQPOs in BHBs (e.g. Middleton \& Done 2010) then a simple inverse-mass relation might be expected for the QPO frequency. Unfortunately, the SMBHs in our sample are of comparable mass (see Table 1), and the present uncertainty in the mass measuring techniques renders any correlation to be dominated by uncertainty.

\section{CONCLUSION}

The search for X-ray QPOs in AGN has been an ongoing challenge within the field for a number of decades (Vaughan \& Uttley 2005) with multiple studies claiming QPO detections (e.g. Vaughan \& Uttley 2005). To-date, only two AGN (non-TDEs) have signals which have been widely accepted as genuine (Gierlinski et al. 2008; Alston et al. 2015). Following Alston et al. (2015) we have analysed energy-dependent periodograms following a robust statistical method and have located significant $(\geq 3 \sigma)$ QPO candidates in five additional AGN.

A number of these candidates appear at the same or similar frequencies across multiple observations, further diminishing the likelihood that they are spurious in nature. However, whilst our approach is robust, there naturally remains uncertainty over the exact shape of the broad-band noise - although we don't anticipate this affecting our results, we will investigate the nature of our candidates in the near future to establish if they demonstrate QPO-like properties.

Our robust detection of QPO candidates using a rigorous approach not only expands the known sample size of such signals, but opens up the possibility of exploring the QPO formation mechanism in new, remarkable detail (Ashton et al. in prep) and implies that new missions (e.g. Athena and STROBE- $X$ ) will more regularly detect such features. 


\section{ACKNOWLEDGEMENTS}

DA and MM thank the anonymous referee for their useful suggestions. This research was possible through an STFC studentship. MM appreciates support via an STFC Ernest Rutherford Fellowship. We thank Will Alston, Simon Vaughan, Phil Uttley, James Leftley, John Paice, Peter Boorman and Adam Hill for helpful discussion. We also reference the use of python libraries which were essential in this analysis: Numpy (van der Walt et al. 2011), Scipy (Virtanen et al. 2020), and Matplotlib (Hunter 2007).

\section{DATA AVAILABILITY}

The data underlying this article are freely accessible in the public HEASARC archives (https://heasarc.gsfc.nasa.gov/).

\section{REFERENCES}

Alston W. N., Vaughan S., Uttley P., 2013, MNRAS, 435, 1511

Alston W. N., Markevičiūtè J., Kara E., Fabian A. C., Middleton M., 2014, MNRAS, 445, L16

Alston W. N., Parker M. L., Markevičiūtė J., Fabian A. C., Middleton M., Lohfink A., Kara E., Pinto C., 2015, MNRAS, 449, 467

Alston W. N., et al., 2019, MNRAS, 482, 2088

Alston W. N., et al., 2020a, Nature Astronomy, p. 2

Alston W. N., et al., 2020b, Nature Astronomy, p. 2

Blaes O. M., Arras P., Fragile P. C., 2006, MNRAS, 369, 1235

Breedt E., et al., 2010, MNRAS, 403, 605

Casebeer D. A., Leighly K. M., Baron E., 2006, ApJ, 637, 157

Churazov E., Gilfanov M., Revnivtsev M., 2001, MNRAS, 321, 759

Coe D., 2009, Fisher Matrices and Confidence Ellipses: A Quick-Start Guide and Software (arXiv: 0906.4123)

Crummy J., Fabian A. C., Gallo L., Ross R. R., 2006, MNRAS, 365, 1067

Denney K. D., et al., 2010, ApJ, 721, 715

Done C., Jin C., 2016, Monthly Notices of the Royal Astronomical Society, 460,1716

Done C., Davis S. W., Jin C., Blaes O., Ward M., 2012a, Monthly Notices of the Royal Astronomical Society, 420, 1848

Done C., Davis S. W., Jin C., Blaes O., Ward M., 2012b, MNRAS, 420, 1848

Fabian A. C., et al., 2009, Nature, 459, 540

Fragile P. C., Blaes O. M., Anninos P., Salmonson J. D., 2007, The Astrophysical Journal, 668, 417

Giacché S., Gilli R., Titarchuk L., 2014, Astronomy \& Astrophysics, 562, A44

Gierliński M., Done C., 2004, MNRAS, 347, 885

Gierlinski M., Middleton M., Ward M., Done C., 2008, Nature, 455, 369

González-Martín O., Vaughan S., 2012, A\&A, 544, A80

Green A. R., McHardy I. M., Done C., 1999, MNRAS, 305, 309

Hunter J. D., 2007, Computing in Science \& Engineering, 9, 90

Ingram A., Done C., 2011, MNRAS, 415, 2323

Ingram A., van der Klis M., Middleton M., Done C., Altamirano D., Heil L., Uttley P., Axelsson M., 2016, MNRAS, 461, 1967

Jin C., Done C., Ward M., 2020, arXiv e-prints, p. arXiv:2005.05857

Kara E., Fabian A. C., Cackett E. M., Steiner J. F., Uttley P., Wilkins D. R., Zoghbi A., 2013, MNRAS, 428, 2795

Kara E., García J. A., Lohfink A., Fabian A. C., Reynolds C. S., Tombesi F., Wilkins D. R., 2017, Monthly Notices of the Royal Astronomical Society, 468,3489

Kara E., et al., 2019, Nature, 565, 198

Liddle A. R., 2007, Monthly Notices of the Royal Astronomical Society: Letters, 377, L74

Markowitz A., Papadakis I., Arévalo P., Turner T. J., Miller L., Reeves J. N., 2007, ApJ, 656, 116
McHardy I. M., Koerding E., Knigge C., Uttley P., Fender R. P., 2006, Nature, 444, 730

McHardy I. M., Arévalo P., Uttley P., Papadakis I. E., Summons D. P., Brinkmann W., Page M. J., 2007, MNRAS, 382, 985

Middleton M., Done C., 2010, MNRAS, 403, 9

Middleton M., Done C., Gierliński M., 2007, MNRAS, 381, 1426

Middleton M., Done C., Ward M., Gierliński M., Schurch N., 2009, MNRAS, 394,250

Middleton M., Uttley P., Done C., 2011, MNRAS, 417, 250

Morgan E. H., Remillard R. A., Greiner J., 1997, ApJ, 482, 993

Motta S. E., 2016, Astronomische Nachrichten, 337, 398

Nowak M. A., 1995, PASP, 107, 1207

Pan H.-W., Yuan W., Yao S., Zhou X.-L., Liu B., Zhou H., Zhang S.-N., 2016, ApJ, 819, L19

Papadakis I. E., Lawrence A., 1995, MNRAS, 272, 161

Papadakis I. E., Brinkmann W., Negoro H., Gliozzi M., 2002, A\&A, 382, L1

Pasham D., Lin D., Saxton R., Jonker P., Kara E., Stone N., Maksym P., Auchettl K., 2019a, BAAS, 51, 27

Pasham D. R., et al., 2019b, Science, 363, 531

Pedregosa F., et al., 2011, Journal of Machine Learning Research, 12, 2825

Peterson B. M., et al., 2000, ApJ, 542, 161

Puchnarewicz E. M., Soria R., 2002, arXiv e-prints, pp astro-ph/0202030

Remillard R. A., McClintock J. E., 2006, Annual Review of Astronomy and Astrophysics, 44, 49

Stella L., Vietri M., Morsink S. M., 1999, The Astrophysical Journal, 524, L63

Timmer J., Koenig M., 1995, A\&A, 300, 707

Ueda Y., Yamaoka K., Remillard R., 2009, ApJ, 695, 888

Uttley P., McHardy I. M., Papadakis I. E., 2002, MNRAS, 332, 231

Uttley P., McHardy I. M., Vaughan S., 2017, A\&A, 601, L1

Vaughan S., 2005, A\&A, 431, 391

Vaughan S., 2010, MNRAS, 402, 307

Vaughan S., Uttley P., 2005, Monthly Notices of the Royal Astronomical Society, 362, 235

Vaughan S., Edelson R., Warwick R. S., Uttley P., 2003, MNRAS, 345, 1271

Vaughan S., Uttley P., Pounds K., Nandra K., Strohmayer T. E., 2011, MNRAS, 413, 2489

Vaughan S., Uttley P., Markowitz A. G., Huppenkothen D., Middleton M. J., Alston W. N., Scargle J. D., Farr W. M., 2016, MNRAS, 461, 3145

Veron-Cetty M. P., Veron P., 2010, VizieR Online Data Catalog, p. VII/258

Virtanen P., et al., 2020, Nature Methods,

Wang, T. Lu, Y. 2001, A\&A, 377, 52

Wilkins D. R., Fabian A. C., 2013, Monthly Notices of the Royal Astronomical Society, 430, 247

Zhang P., Zhang P.-f., Yan J.-z., Fan Y.-z., Liu Q.-z., 2017, The Astrophysical Journal, 849, 9

Zhang P.-f., Zhang P., Liao N.-h., Yan J.-z., Fan Y.-z., Liu Q.-z., 2018, ApJ, 853, 193

van der Klis M., 1988, in Timing Neutron Stars, eds. H. Ogelman and E.P.J. van den Heuvel. NATO ASI Series C, Vol. 262, p. 27-70. Dordrecht: Kluwer, 1988.. pp 27-70

van der Walt S., Colbert S. C., Varoquaux G., 2011, Computing in Science Engineering, 13, 22

This paper has been typeset from a $\mathrm{T}_{\mathrm{E}} \mathrm{X} / \mathrm{L} \mathrm{T} \mathrm{T} \mathrm{X}$ file prepared by the author. 
Table 2. Details of the observations used in this study. The columns indicate: (1) Active Galactic Nuclei in the sample, (2): Observation ID, (3): The total EPIC-PN exposure time, (4): The length of the longest flare-less segment within the observation. The full table is available online.

\begin{tabular}{cccc}
\hline AGN & Obs ID & Exposure Time $(\mathrm{ks})$ & Maximum Flareless Exposure $(\mathrm{ks})$ \\
\hline PG 0003+199 & 0101040101 & 31.60 & 23.44 \\
PG 0003+199 & 0306870101 & 132.70 & 132.70 \\
PG 0003+199 & 0510010701 & 16.70 & 16.70 \\
PG 0003+199 & 0600540501 & 80.70 & 34.96 \\
PG 0003+199 & 0600540601 & 130.40 & 130.40 \\
PG 0003+199 & 0741280201 & 137.40 & 137.40 \\
PG 0050+124 & 0110890301 & 20.00 & 20.00 \\
PG 0050+124 & 0300470101 & 82.90 & 24.00 \\
PG 0050+124 & 0743050301 & 20.40 & 2.37 \\
PG 0050+124 & 0743050801 & 20.40 & 20.40 \\
PG 0157+001 & 0101640201 & 11.20 & 10.19
\end{tabular}

